NIST Special Publication 250-93

\title{
NIST Calibration Services for Spinning Rotor Gauge Calibrations
}

Robert F. Berg James A. Fedchak

This publication is available free of charge from:

http://dx.doi.org/10.6028/NIST.SP.250-93

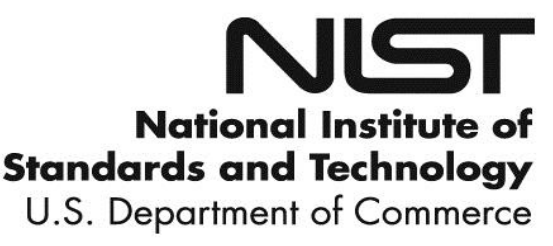


NIST Special Publication 250-93

\title{
NIST Calibration Services for Spinning Rotor Gauge Calibrations
}

\author{
Robert F. Berg \\ James A. Fedchak \\ Sensor Science Division \\ Physical Measurement Laboratory
}

This publication is available free of charge from:

http://dx.doi.org/10.6028/NIST.SP.250-93

May 2015

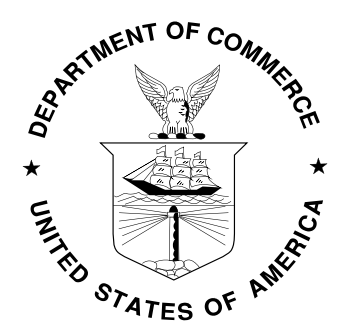

U.S. Department of Commerce

Penny Pritzker, Secretary

National Institute of Standards and Technology Willie May, Under Secretary of Commerce for Standards and Technology and Director 
Certain commercial entities, equipment, or materials may be identified in this document in order to describe an experimental procedure or concept adequately. Such identification is not intended to imply recommendation or endorsement by the National Institute of Standards and Technology, nor is it intended to imply that the entities, materials, or equipment are necessarily the best available for the purpose.

National Institute of Standards and Technology Special Publication 250-93 Natl. Inst. Stand. Technol. Spec. Publ. 250-93, 42 pages (May 2015) CODEN: NSPUE2

This publication is available free of charge from: http://dx.doi.org/10.6028/NIST.SP.250-93 


\section{NIST Special Publication 250-93 2014 NIST Calibration Services For Spinning Rotor Gauge Calibrations}

Robert F. Berg and James A. Fedchak

\section{Contents}

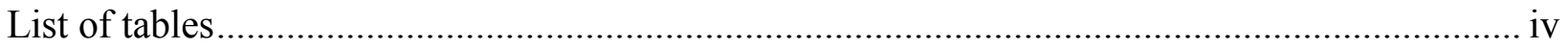

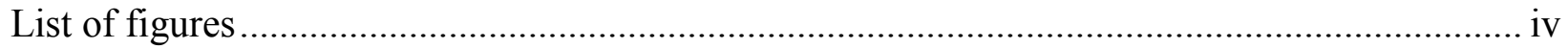

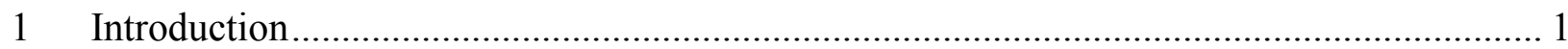

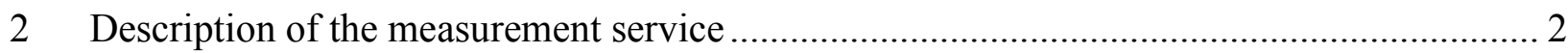

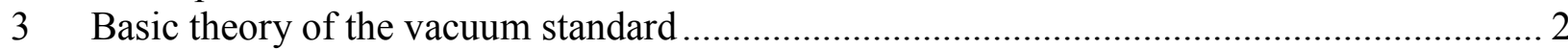

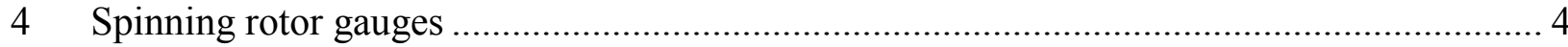

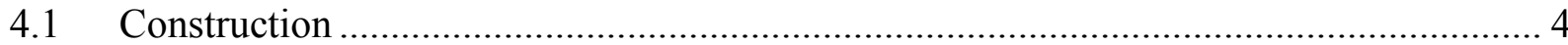

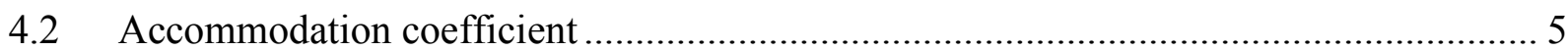

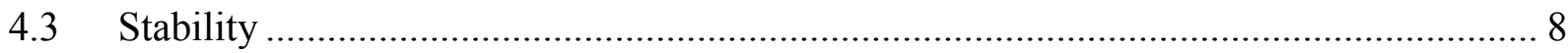

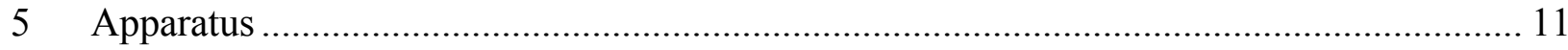

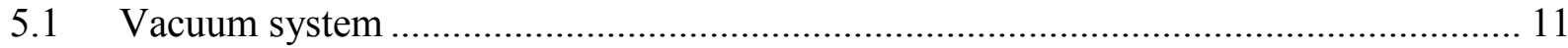

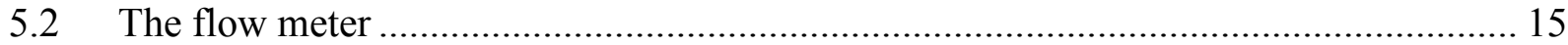

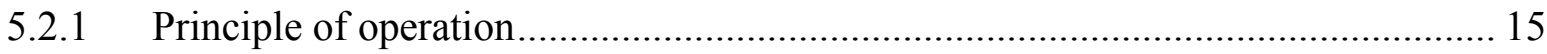

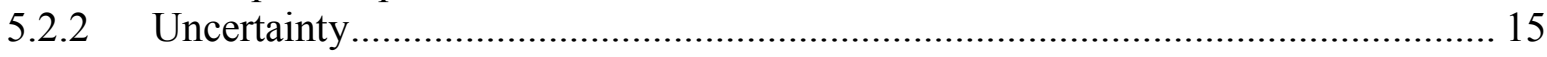

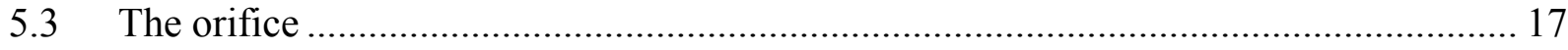

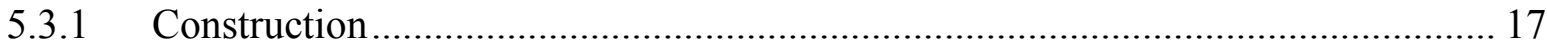

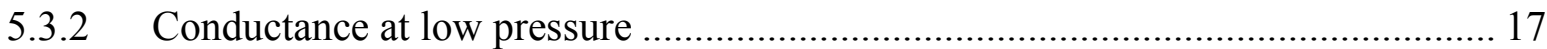

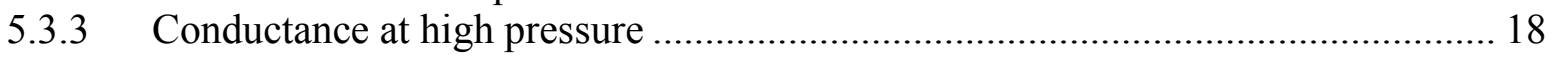

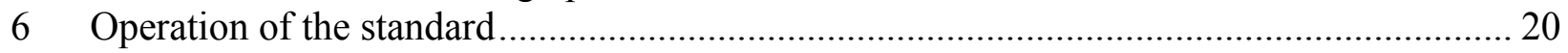

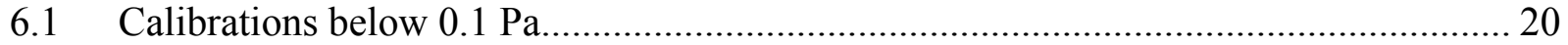

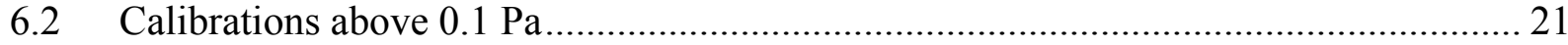

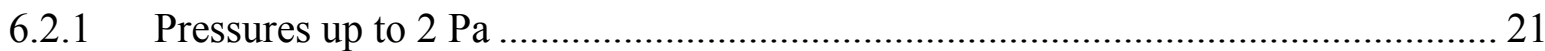

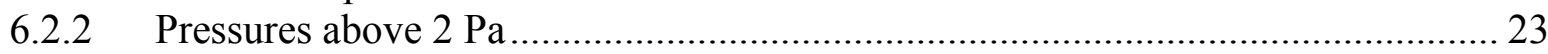

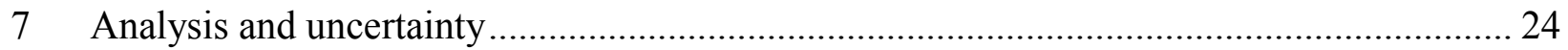

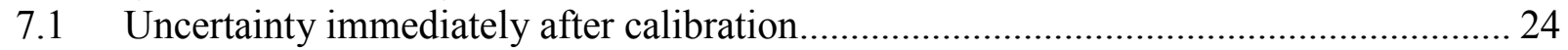

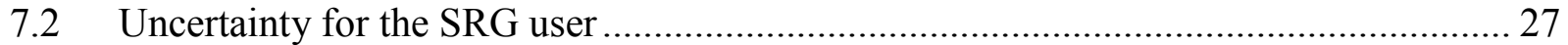

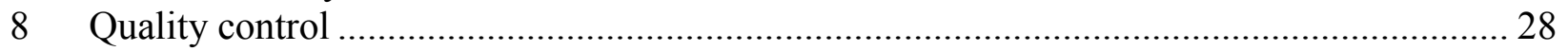

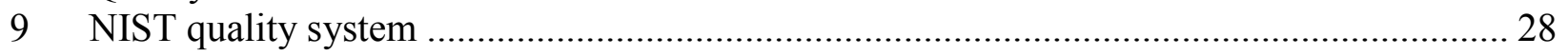

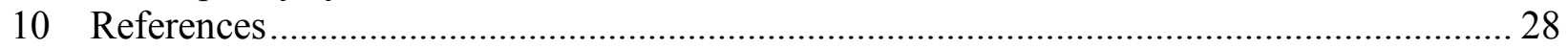




\section{List of tables}

1. Changes in accommodation coefficient for NIST check standards and customer rotors ....8

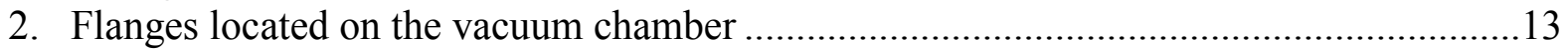

3. Contributions to the Type B uncertainty of the flow meters ........................................16

4. Molecular flow characteristics of the two orifices and their standard uncertainties .........18

5. The first-order pressure coefficient $\alpha_{1}\left(\mathrm{~Pa}^{-1}\right)$ and its standard uncertainty .....................19

6. Measurements of the polynomial coefficients in Eq. (14) for the $2 \mathrm{~mm}$ orifice................19

7. Relative standard uncertainties and weighted uncertainty contributions..........................26

\section{List of figures}

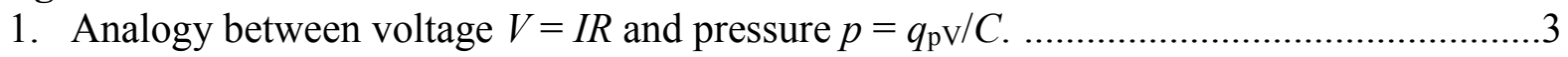

2. Cross section through an SRG suspension head and thimble. .....................................5

3. Comparison of SRG pressure readings to a calibrated CDG. .......................................

4. Change in the accommodation coefficient between SRG calibrations. ............................. 9

5. History of the accommodation coefficient for three NIST check standards. ....................9

6. History of the accommodation coefficient for two SRG rotors. ......................................10

7. Accommodation coefficient as a function of head tilt. ..................................................11

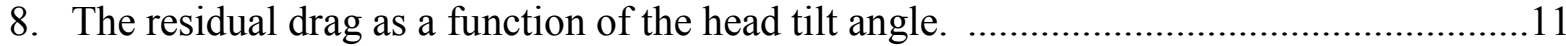

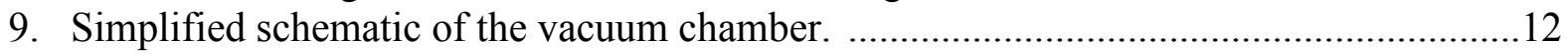

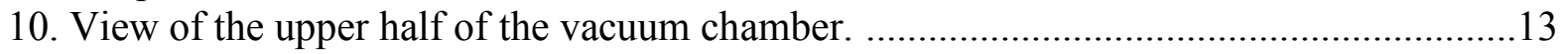

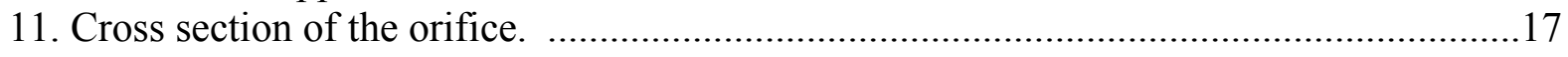

12. The pressure ratio for nitrogen flow through the $11 \mathrm{~mm}$ orifice. ...................................19

13. Results for the accommodation coefficient of a customer rotor in nitrogen. ..................22

14. The accommodation coefficient as a function of pressure for 9 rotors. ..........................23 


\section{Introduction}

"Vacuum" covers an enormous range of pressures below one atmosphere $\left(10^{5} \mathrm{~Pa}\right)$. Uses of vacuum vary from building infiltration tests (only $50 \mathrm{~Pa}$ below one atmosphere) to high energy particle accelerators $\left(10^{-9} \mathrm{~Pa}\right)$. The uncertainty required of the corresponding vacuum measurements also varies widely. For many industrial applications, a $10 \%$ uncertainty of the pressure reading is often sufficient, although the reproducibility requirement can sometimes be as small as $1 \%$. Some scientific applications require an uncertainty better than $1 \%$.

Most vacuum gauges are calibrated at the factory by comparing the newly manufactured gauge to a working standard gauge. Typically, the working standard gauge is calibrated against a second, more accurate standard. At the top of the calibration chain lies a primary standard that uses well-understood physical principles to produce a known pressure. Several such primary standards at the National Institute of Standards and Technology (NIST) provide calibrations for a variety of vacuum gauges, from one atmosphere down to $10^{-7} \mathrm{~Pa}$.

A spinning rotor gauge (also called SRG, molecular drag gauge, or gas friction gauge) is rarely used for control in industrial processes. This is mainly because the large residual drag (zero reading) of the SRG limits its useful range to pressures above about $10^{-5} \mathrm{~Pa}$. Although the residual drag is stable over the short term, it must be re-measured every time the SRG is resuspended, and it can vary from $10^{-5} \mathrm{~Pa}$ to $10^{-3} \mathrm{~Pa}$. Therefore, the vacuum system must achieve a low base pressure to determine the residual drag before the SRG can be used, and the base pressure usually needs to be verified with a second gauge, typically an ionization gauge. This makes it difficult or impossible to use an SRG to determine the pressure during an initial vacuum system pump-down. In addition, the response is rather slow for process control; a typical integration time for an SRG is $30 \mathrm{~s}$. Since an SRG has excellent short-term and long-term stability, ruggedness, and predictability, it is most often used as a transfer standard over the pressure range of $10^{-4} \mathrm{~Pa}$ to $10^{-1} \mathrm{~Pa}$, and is often the basis of secondary standards for the calibration of other vacuum gauges or for the generation of a known gas flow. At lower pressures, ionization gauges are typically used as transfer standards, and at higher vacuum pressures one typically uses capacitance diaphragm gauges or resonant silicon gauges as transfer standards; NIST has primary standards that covers calibration of these gauges, but those services is outside the scope of this publication.

This Special Publication describes the calibration services for spinning rotor gauges in the pressure range of $10^{-4} \mathrm{~Pa}$ to $1 \mathrm{~Pa}$. Two calibration services are offered for SRGs: Standard Calibrations, which include only $\mathrm{N}_{2}$ pressures $<0.1 \mathrm{~Pa}$, and Special Calibrations, which cover special customer requests, such as using other gases or pressures.

The Standard Calibration Service is performed using the medium-range vacuum standard. That standard, in addition to one that is used to calibrate ionization gauges, was preceded by a similar standard constructed in the 1980s [1,2]. A few years later the present two standards were constructed and installed in Building 220, and in 2004 they were moved to Building 218. Studies of the medium-range standard are described in a series of internal reports [3-10]. In some rare situations, the Special Calibration Service for SRGs may require vacuum standards other than the medium-range vacuum standard. Typically, this would be another high vacuum standard that uses the dynamic expansion technique described in Refs. [1,2], which would have a similar 
uncertainty budget. This document describes only the medium-range standard that is used for all of the SRG Standard Calibrations and the majority of the SRG Special Calibrations.

Two books with useful general information about vacuum gauges and calibration methods are Refs. [11] and [12].

\section{Description of the measurement service}

NIST provides the following calibration services for spinning rotor gauges:

Standard Calibrations (C-test)

- Nitrogen gas at one pressure below $0.1 \mathrm{~Pa}$, typically $0.02 \mathrm{~Pa}$.

Special Calibrations (S-test)

- An inert gas other than nitrogen at one pressure below $0.1 \mathrm{~Pa}$.

- An inert gas or $\mathrm{N}_{2}$ at pressures above $0.1 \mathrm{~Pa}$.

- Other special circumstances or tests.

The NIST Thermodynamic Metrology Group conducts SRG calibrations for $\mathrm{N}_{2}$ at one pressure below $0.1 \mathrm{~Pa}$ in batches one or two times per year. Arranging an SRG calibration has the following steps.

1. Contact the appropriate technical staff member of the NIST Thermodynamic Metrology Group to discuss the schedule and other details of the service. The appropriate person is identified on the calibration pages on NIST's external website.

2. The technical staff member will arrange for a Pro Forma Invoice to be signed and returned to NIST. This must be completed before a calibration is performed.

3. Send a purchase order to NIST. The fee for a standard calibration is updated annually on NIST's external website. The fee for a Special Calibration is done at cost and will depend on the nature of the special test.

4. Send the SRG equipment to the technical contact. In principle, only the rotor needs to be sent, but customers are encouraged to send the suspension head and the controller.

The appendix contains an example of an SRG calibration report.

\section{Basic theory of the primary vacuum standard}

The primary standard utilizes the "dynamic expansion" technique, in which the calibration or standard pressure $p_{s t d}(\mathrm{~Pa})$ is generated by gas flowing at a known throughput $q_{p V}\left(\mathrm{~Pa} \mathrm{~m}^{3} \mathrm{~s}^{-1}\right)$ through an orifice of known conductance $C\left(\mathrm{~m}^{3} \mathrm{~s}^{-1}\right)$. The pressure drop across the orifice is given by:

$$
\Delta p=\frac{q_{p V}}{C} .
$$

If the downstream pressure is negligible compared to the upstream pressure, then $\Delta p=p_{\text {std }}$. An accurate absolute flow meter generates the gas flow, and a gas-kinetic theory calculation based on the orifice dimensions gives the conductance of the orifice. The analogy with Ohm's law outlined in Figure 1 may be helpful. 

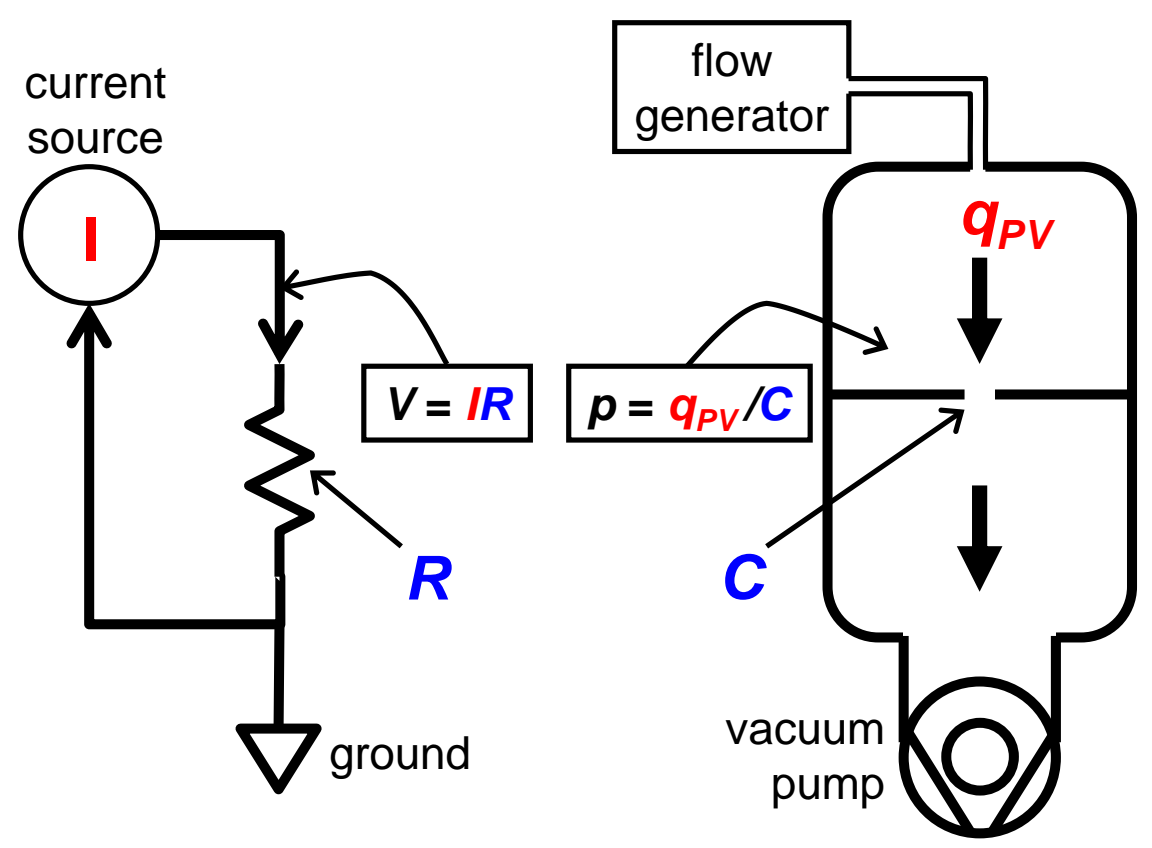

Figure 1. The analogy between the voltage $V=I R$ generated by an electrical current $I$ flowing through a resistance $R$, and the pressure $p=q_{p} / C$ generated by a gas flow $q_{p V}$ through an orifice of conductance $C$.

Another method for calibrating vacuum gauges is the "static expansion" technique, in which the calibration pressure is generated by filling a small volume $V_{1}$ to a known pressure $p_{1}$ and then expanding to a larger volume $V_{2}$, so that $p=\left(V_{1} / V_{2}\right) p_{1}$. Dynamic expansion offers several advantages over static expansion for generating gas pressures in the high vacuum regime. First, stable pressures are readily generated for long intervals. Second, because the flow is steady over a long interval, transient behavior associated with adsorption and desorption (outgassing) either have a negligible effect on the calculated standard pressure or produce a small and easily characterized contribution to the uncertainty budget. Third, temperature stability is less of a concern [13-14]. Finally, the only dimensions which need to be characterized are those of the orifice and the piston, and these are not dependent on the position of any valve. On the other hand, static expansion systems offer a clear advantage at pressures above $1 \mathrm{~Pa}$, where outgassing and adsorption become small uncertainty components but, in the dynamic expansion system, transition flow corrections to the orifice conductance become a large uncertainty component. Presently, NIST only uses the dynamic expansion technique for SRGs, and the majority of SRG calibrations are for $p<100 \mathrm{mPa}$.

In practice, the pressure drop across the orifice is not solely determined by the upstream pressure. The pressure in the upper chamber $p_{\text {std }}$ is the sum of the pressure in the lower chamber $p_{\mathrm{L}}$ and the pressure drop $q_{p} / C$ :

$$
p_{s t d}=p_{L}+\frac{q_{p V}}{C} .
$$

It is difficult to measure $p_{\mathrm{L}}$ absolutely because this would require a gauge calibrated below the range of pressures generated by the standard. However, one can use an SRG to accurately measure the pressure ratio, 


$$
R_{P}=\frac{p_{U}}{p_{L}} .
$$

Here we use $p_{U}$ instead of $p_{s t d}$ since $p_{U}$ represents the measured upstream pressure and not the calculated standard pressure. Since the SRG decrement is linear for $p<0.1 \mathrm{~Pa}, R_{P}$ does not depend on the SRG calibration. From Eqs. (2) and (3) one obtains

$$
p_{s t d}=\frac{q_{p V}}{C}\left(\frac{R_{P}}{R_{P}-1}\right) .
$$

Eq. (4) is the basic equation for the standard at low pressures. Calibrations of high vacuum gauges are performed by attaching the gauge to the upper chamber and comparing $p_{\text {std }}$ with the gauge reading.

For the two orifices used in the standard, the pressure ratio is always greater than 20 , and the uncertainty contribution of $R_{P}$ is reduced by $1 / R_{P}$; therefore $p_{\text {std }}$ is insensitive to a small error or bias in $R_{P}$. Furthermore, $R_{P}$ depends only on the orifice conductance and the speed of the turbo pump, both of which are effectively independent of pressure below $0.1 \mathrm{~Pa}$.

\section{Spinning rotor gauges}

This section discusses the construction, accommodation coefficient, and stability of spinning rotor gauges.

\subsection{Construction}

The heart of a spinning rotor gauge is a magnetically suspended steel sphere (rotor), usually a steel ball bearing nominally $4.5 \mathrm{~mm}$ in diameter. A combination of permanent magnets and controlled electromagnets keeps the rotor suspended within a cylindrical tube (thimble) that is attached to the vacuum chamber. Several sets of wire coils used for vertical and horizontal stabilization, driving the rotor, and sensing the rotation Drive coils cause the sphere to rotate until the frequency is approximately $f=410 \mathrm{~Hz}$. After the drive coils are turned off, gas molecules that collide with the rotor cause the rotation frequency to decrease. The rate of decrease $d f / d t$ is detected by sensing coils that couple to the magnetic moment of the steel sphere. Since that moment is typically not aligned with the sphere's vertical rotation axis, the resulting signal oscillates at frequency $f$. Figure 2 shows the cross section of a typical suspension head and thimble.

The thimble containing the rotor has a negligible effect on the SRG's ability to measure pressure below $0.1 \mathrm{~Pa}$, and a properly operating controller and suspension head have no effect on the pressure accuracy of the SRG. Thus, an SRG calibration depends only on the rotor. It must possess both a magnetic moment and an asymmetry in its inertia tensor that is small but nonzero; any slight density homogeneity or asphericity will create the asymmetry. The suspending magnetic field produces a torque that tends to align the magnetic moment with the magnetic field. At the same time, the moment of inertia produces a torque that tends to align the axis of the largest principal moment with the rotation axis. Since the magnetic moment and the principle axis will not, in general, be aligned, neither will be perfectly aligned with the rotation axis. This misalignment allows the detection of the rotor's rotation because it is the rotating magnetic moment that produces the signal in the pickup coil. (The signal would be zero if the magnetic 
moment were aligned with the rotation axis.) However, if the principle moment of inertia is too large, the residual drag will have a large frequency dependence. This can be taken into account mathematically, but it may reduce accuracy at lower pressures.

In principle, any diameter ball bearing may be used as a rotor but, in practice, present-day commercially available SRGs typically use steel ball bearings with diameters of $4.5 \mathrm{~mm}$, and sometimes $4.76 \mathrm{~mm}$. Rotors may be either smooth or etched and are commonly made of either chrome steel (such as SAE 52100) or 400 series stainless steel (such as 440C). Invar rotors have also been successfully used, but are not commonly used by customers of the calibration service.

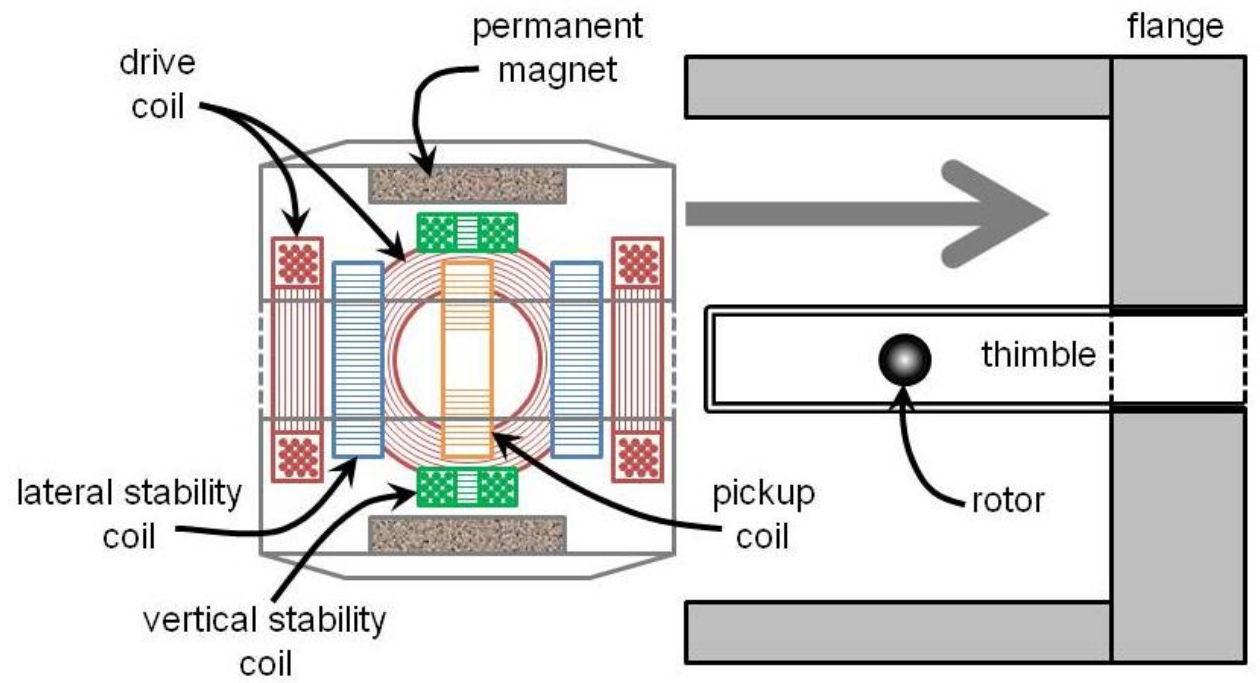

Figure 2. Cross section through an SRG suspension head and thimble (approximate scale).

\subsection{Accommodation coefficient}

Even without calibration, one can use a spinning rotor gauge to obtain a pressure reading that is moderately accurate. In the molecular flow regime, the relation between the SRG pressure reading $p_{\mathrm{SRG}}$ and the rotor deceleration rate is

$$
\begin{aligned}
p_{\mathrm{SRG}} & =\frac{\pi \bar{v} \rho d}{20}\left(D C R-D C R_{0}-2 \alpha \frac{d T}{d t}\right) \\
& =\left(\frac{2 \pi R T}{M}\right)^{1 / 2} \frac{\rho d}{10}\left(D C R-D C R_{0}-2 \alpha \frac{d T}{d t}\right)
\end{aligned}
$$

where 


$$
\begin{aligned}
R & =\text { universal gas constant }\left(8.314772 \mathrm{~J} \mathrm{~mol}^{-1} \mathrm{~K}^{-1}\right) \\
\bar{v} & =\text { mean thermal velocity of the gas }(\mathrm{m} / \mathrm{s}) \\
T & =\text { gas temperature }(\mathrm{K}) \\
M & =\text { gas molar mass }\left(\mathrm{kg} \mathrm{mol}^{-1}\right) \\
\rho & =\text { rotor density }\left(\mathrm{kg} \mathrm{m}^{-3}\right) \\
d & =\text { rotor diameter }(\mathrm{m}) \\
D C R & =-(d f / d t) / f=\text { normalized rotor deceleration rate }\left(\mathrm{s}^{-1}\right) \\
D C R_{0} & =\text { offset }=\text { value of } D C R \text { measured at base pressure }(\mathrm{Pa}) \\
\alpha & =\text { thermal linear expansion coefficient }\left(\mathrm{K}^{-1}\right)
\end{aligned}
$$

As will be explained in Section 4.3, the last term in Eq. (5) describes the thermal expansion of the rotor and is unnecessary in a normal standard NIST calibration. The SRG pressure reading $p_{\text {SRG }}$ differs from the true pressure $p_{\text {true }}$ by the accommodation coefficient:

$$
p_{\text {true }}=\frac{1}{\sigma} p_{S R G} \text {. }
$$

$p_{\text {true }}$ is determined by the pressure standard, $p_{\text {true }}=p_{\text {std }}$, and the accommodation coefficient is defined by the ratio

$$
\sigma=\frac{p_{\mathrm{SRG}}}{p_{\text {std }}}
$$

Calibrating a spinning rotor gauge is equivalent to determining $\sigma$. For a perfectly smooth ideal spherical rotor, $\sigma=\sigma_{t}$, where $\sigma_{t}$ is the tangential momentum accommodation coefficient representing the fraction of tangential momentum that a typical gas molecule acquires from the rotor during a single collision. Perfect momentum accommodation means that $\sigma_{t}=1$ and the gas molecule leaves the rotor surface with a tangential velocity component identical to the surface velocity of the rotating sphere. Thus $0<\sigma_{t} \leq 1$. However, technical materials have a surface roughness that affects the angle at which the gas molecules leave the surface. If the surface roughness is taken into account, it can be shown that an effective accommodation coefficient can have a value as high as $\sigma_{e f f}=1.27$ [1616]. In calibrations, it is the effective accommodation coefficient, taking into account both the momentum accommodation and surface roughness, which is determined. In addition, nominal values of $d$ and $\rho$ are typically used with no associated uncertainties, and so $\sigma_{\text {eff }}$ also includes the difference between these nominal values and the true values. For the remainder of this document, we take $\sigma=\sigma_{\text {eff. The effective accommodation }}$ coefficient usually falls within the range $\sigma=1.00 \pm 0.03$ [17]. Thus, the spinning rotor gauge can be considered a primary pressure gauge with a standard uncertainty of approximately $3 \%$.

Part of the normalized rotor deceleration rate $(d f / d t) / f$ is an offset due to eddy currents induced within the sphere or within the surrounding metal parts. This offset, also called "residual drag" or "vacuum decrement", is equivalent to an apparent pressure in the range from $10^{-5} \mathrm{~Pa}$ to $10^{-3} \mathrm{~Pa}$. The size and frequency dependence of the offset is influenced by the angle between the principle inertial axis and the magnetic moment [18]. 
Above $0.1 \mathrm{~Pa}$, the decrement is no longer a linear function of pressure and Eq. (5) is no longer valid. This is not an issue for Standard Calibrations (C-test) since $\sigma$ is determined at a single pressure $<0.1 \mathrm{~Pa}$, nominally $20 \mathrm{mPa}$. However, calibrations at higher pressures may be provided as part of a Special Calibration. Between $0.1 \mathrm{~Pa}$ and $2 \mathrm{~Pa}$, Eq. (5) may be applied if the accommodation coefficient is modelled as $\sigma_{\text {eff }}(p)=a+b p$, with $a=\sigma_{\text {eff }}(p \leq 0.1 \mathrm{~Pa})$ [19]. A minimum of two pressure points are needed to determine the slope $b$ for a calibration in this range. Above $1 \mathrm{~Pa}$, using an SRG as a transfer standard is not recommended. The rapid deceleration of the rotor causes heating due to the eddy currents and leads to temperature instability of the rotor. Other high-quality gauges, such as capacitive diaphragm gauges, are more useful as a transfer standard at higher pressures.

SRG controllers can display decrement or pressure. For calibrations, NIST always reads the decrement and uses Eq. (5) to determine the gauge pressure. This avoids potential errors due to controller setup and adds clarity to the analysis. Users of SRGs often find it convenient to program a data set into the controller that contains $\sigma$ and the other parameters in Eq. (5), including the residual drag. Above $0.1 \mathrm{~Pa}$, the data set must include the gas viscosity so that the controller can perform a linearization routine to determine the pressure [20,21]. Tests at NIST were conducted in 2009 to compare the $\mathrm{N}_{2}$ pressure readings from three models of MKS SRG controllers to a CDG reading; the results are shown in Figure 3. Up to a pressure of $800 \mathrm{mPa}$, the SRG readings agree with the CDG readings to within $0.1 \%$ and are within the uncertainty of the comparison. At $1 \mathrm{~Pa}$, the SRG and CDG disagree by more than $0.4 \%$, and at $10 \mathrm{~Pa}$ (not shown in the figure) the CDG and SRG disagreed by more than $3 \%$. Therefore it seems that SRG controllers do a reasonable job of linearizing the pressure up to about $1 \mathrm{~Pa}$, which confirms earlier work at NIST [21]. However, for metrology work the controller linearization routine cannot be trusted since the proprietary algorithms are unknown to the user, and they may differ among controllers. Indeed, a 1993 study [21] found errors in two brands of SRG controllers. For users desiring accurate metrological pressure measurements above $0.1 \mathrm{~Pa}$, we advise the 2 point calibration scheme described above. 


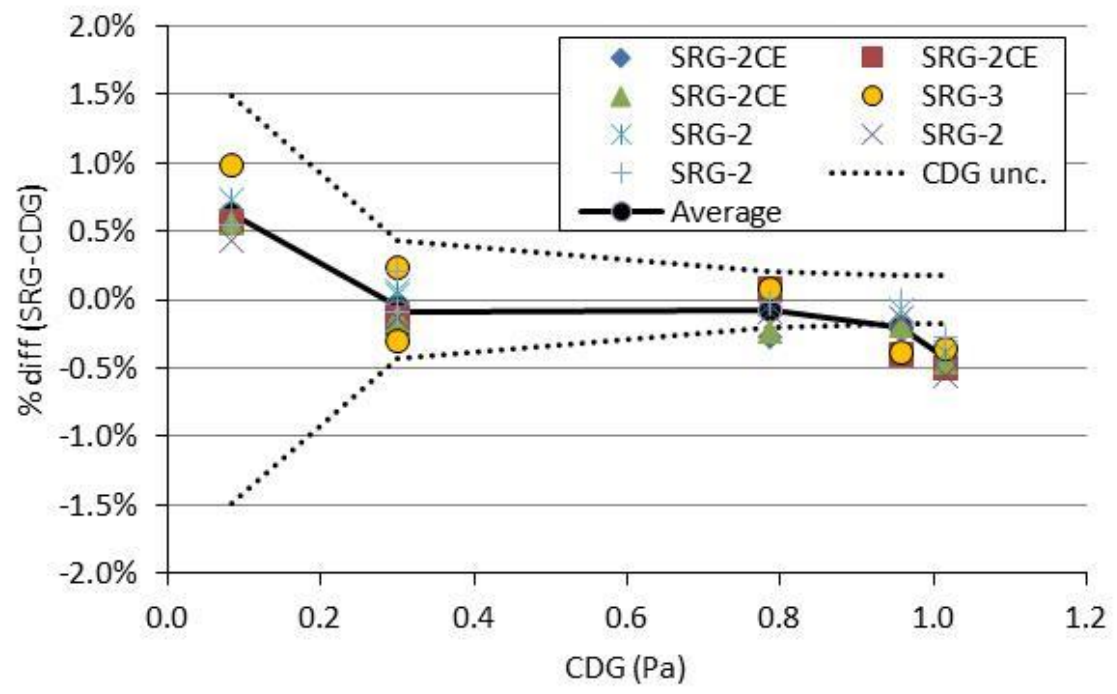

Figure 3. Comparison of SRG pressure readings to a calibrated CDG. The SRG pressure readings agree well with the CDG to about $0.8 \mathrm{~Pa}$. At $1 \mathrm{~Pa}$, the CDG and SRG disagree by about $0.4 \%$. At $10 \mathrm{~Pa}$ (data not shown), the disagreement was more than $3 \%$.

\subsection{Stability}

The offset or residual drag is measured at a pressure that is comparable to or lower than the gauge's reproducibility. For the majority of rotors, the mean reproducibility of the offset is approximately $7 \times 10^{-10} \mathrm{~s}^{-1}(k=1)$ and can range from $2 \times 10^{-10} \mathrm{~s}^{-1}$ to $2 \times 10^{-9} \mathrm{~s}^{-1}$, which corresponds to a pressure range of $4 \times 10^{-7} \mathrm{~Pa}$ to $4 \times 10^{-6} \mathrm{~Pa}$. Factors affecting the short-term reproducibility of the offset or of the decrement measurement reading are vibration, temperature changes, signal strength, and errors of the timing circuitry. Vibration is the largest effect. Temperature changes affect the SRG through thermal expansion of the rotor. For a typical steel rotor of $d=4.5 \mathrm{~mm}, \rho=7.7 \mathrm{~g} / \mathrm{cm}^{3}$, and $\alpha \sim 5 \times 10^{-5} / \mathrm{K}$, a temperature change of $0.25 \mathrm{~K} / \mathrm{h}$ gives a $0.1 \%$ change in the decrement at $20 \mathrm{mPa}$. The NIST laboratory temperature is controlled to better than $0.25 \mathrm{~K} / \mathrm{h}$, and the typical temperature changes of the vacuum chamber much smaller; therefore temperature changes are not a relevant factor in NIST SRG calibrations.

Table 1. Statistics of change in accommodation coefficient for NIST check standards and customer rotors(reproduced from Ref [22]).

\begin{tabular}{lcc} 
& check standards & customers \\
\hline Number of gauges & 3 & 70 \\
Number of samples & 69 & 155 \\
Mean time between calibrations & 0.50 years & 2.53 years \\
Mean of absolute change & $0.16 \%$ & $0.94 \%$ \\
Median of absolute change & $0.10 \%$ & $0.72 \%$ \\
Percent of samples with change $<0.1 \%$ & $49 \%$ & $10 \%$ \\
Percent of samples with change $<0.5 \%$ & $94 \%$ & $40 \%$ \\
Percent of samples with change $<1 \%$ & $100 \%$ & $63 \%$ \\
Percent of samples with change $<2 \%$ & $100 \%$ & $88 \%$ \\
\hline
\end{tabular}




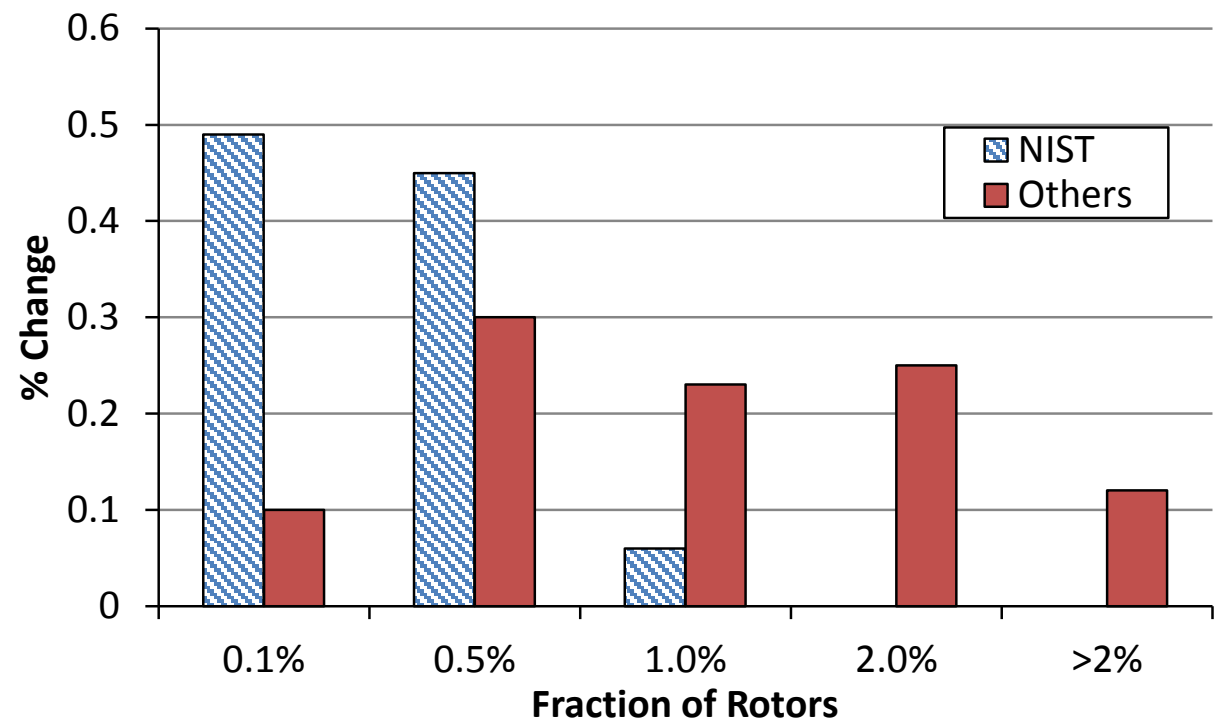

Figure 4. Histogram showing percent change in the accommodation coefficient between SRG calibrations.

With careful use, the accommodation coefficient of most SRGs will change no more than $2 \%$ over a 2-year period. This can be seen in the historical data from NIST's SRG calibration database that are summarized in Table 1 and Figure 4 . Figure 5 shows the accommodation coefficient $\sigma$ measured for three NIST check standards over a 20 year interval. The change of $\sigma$ between calibrations was typically less than $0.2 \%$, and, with one exception, never exceeded $0.5 \%$. In contrast, Figure 6 shows the calibration history for two customer rotors. The rotor from customer A had a time dependence that was much larger than for customer B. In addition, it had corrosion on $1 / 4$ of its area as well as heavy scratches, which suggests that its use was less than careful.

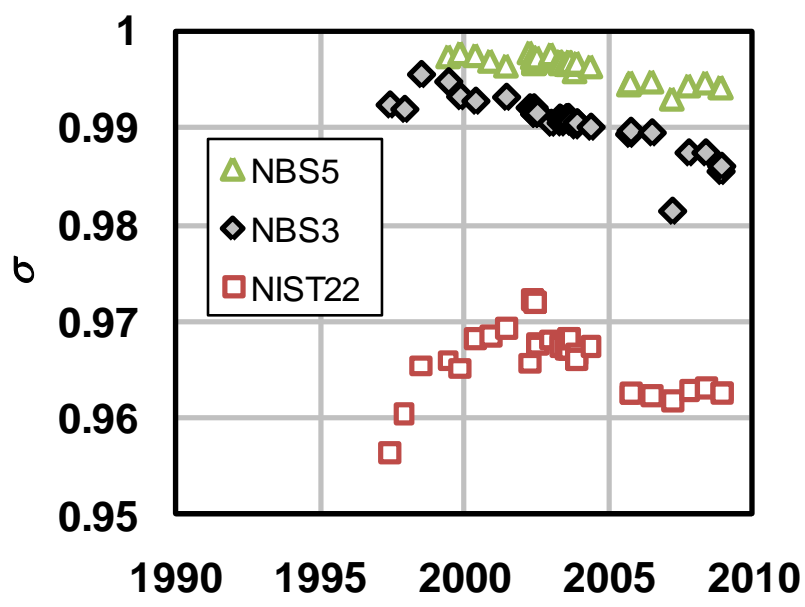

Figure 5. History of the accommodation coefficient $\sigma$ for three NIST check standards. 


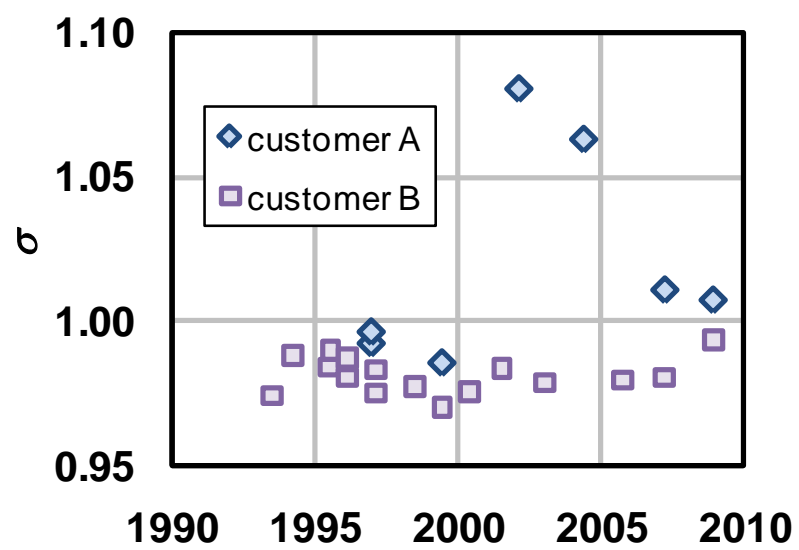

Figure 6. History of the accommodation coefficient $\sigma$ for two SRG rotors. The vertical scale is 3 times larger than for Figure 5.

Chang and Abbott [22] studied possible causes for the irreproducibility of SRGs, including baking, turning off the suspension after decelerating the rotor, and turning off the suspension without fully decelerating ("crashing") the rotor. They found that "contact tests", which included washing with rotor with acetone and ethanol, violently shaking the rotor, and removing and reinstalling the head, produced the largest changes in accommodation coefficient, between $0.1 \%$ and $0.4 \%$ in their study. Perhaps one of the more surprising results of the study was that crashing a spinning rotor produced less than a $0.1 \%$ change, which was less than the change produced by simply removing and reinstalling the head. Chang and Abbott explained their results by postulating that (1) the surface roughness may not be uniform, so a small change in the rotation axis could change the surface-averaged accommodation coefficient, and (2) that removing and re-installing the head would not preserve the axis of rotation. It is possible that the initial surface conditions could play a role in the crash tests. For example, rotors that are initially smooth and free of scratches may perform differently than those that are rough. Experience in other labs [23] suggests that crash tests may, in some cases, have a larger effect than reported in Chang and Abbott [22]. In any case, crashing the rotor should be avoided as it may change the accommodation coefficient.

It is common practice to mount spinning rotor gauges such that the head tilt is less than $2^{\circ}$ from vertical. This is easily accomplished using a standard bubble level. In reality, the head tilt can be much greater than $2^{\circ}$ without affecting the accommodation coefficient or stability. Figure 7 shows a plot of $\sigma$ as a function of head tilt. Over a range of $8^{\circ}$ the accommodation coefficient varied by less than $0.04 \%$. On the other hand, the residual drag had a strong dependence on head tilt, as shown in Figure 8 . A tilt of $2^{\circ}$ caused a negligible change, but at tilt of $4^{\circ}$ increased the residual drag by $1 \times 10^{-6}$. Because the residual drag is always measured and subtracted from the total decrement, keeping the tilt to within $2^{\circ}$ is good practice but not critical. The 2013 data shown in Figure 7 and Figure 8 confirm earlier work by Lindenau, Fremerey, and Witthauer [24]. 


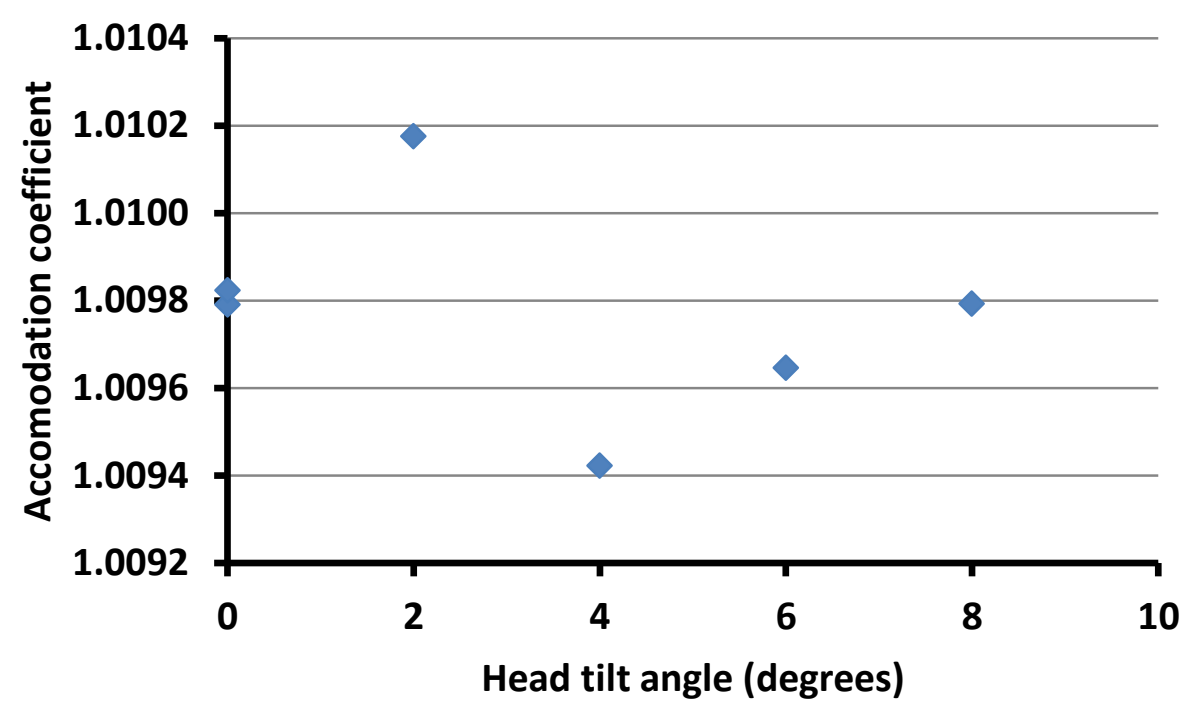

Figure 7. Accommodation coefficient as a function of head tilt

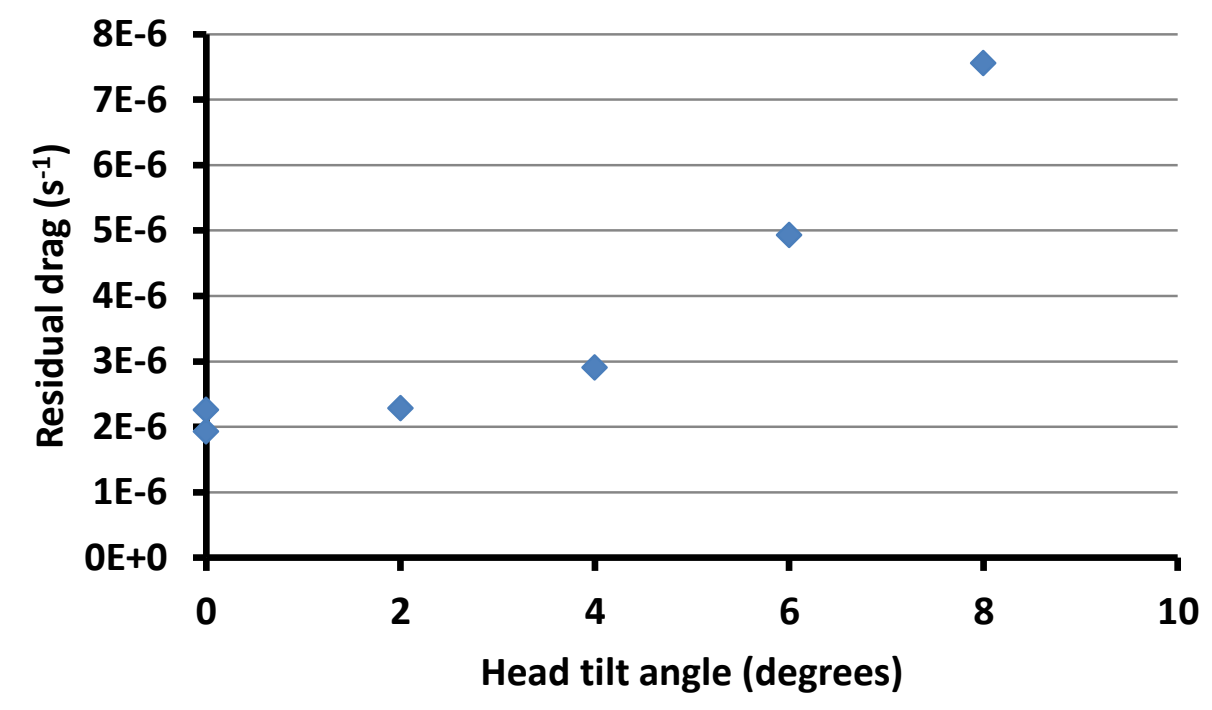

Figure 8. The residual drag (RD) as a function of the head tilt angle.

\section{Apparatus}

This section discusses the three main parts of the apparatus: the vacuum chamber, the flow meter, and the orifice.

\subsection{Vacuum system}

The vacuum system was designed to maintain a low background pressure, provide room for mounting many vacuum gauges, expose the gauges to a uniform pressure, and ensure that the orifice conductance calculation is valid. The main components are the chamber support, the upper half of the chamber, the orifice, the lower half of the chamber, the vacuum pump, and the external plumbing. 
An aluminum frame supports the chamber and a surrounding table, which is made of ceramic fiber board covered by sheet aluminum. A large oven may be placed on the table that encloses the chamber for baking. Baking is not a part of the Standard Calibration Service.

Figure 9 is a schematic of the vacuum system, and Figure 10 is a photograph of the upper half of the vacuum chamber with the top flange removed (14.25 inch CF). The two halves of the chamber are separated by a horizontal plate that holds the orifice. Each half is made of type 304 stainless steel and is $30 \mathrm{~cm}$ in diameter and $34 \mathrm{~cm}$ long. Table 3 gives the typical functions of the flanges attached to the chamber. The blank flanges on the upper chamber allow as many as 14 SRGs to be calibrated at once.

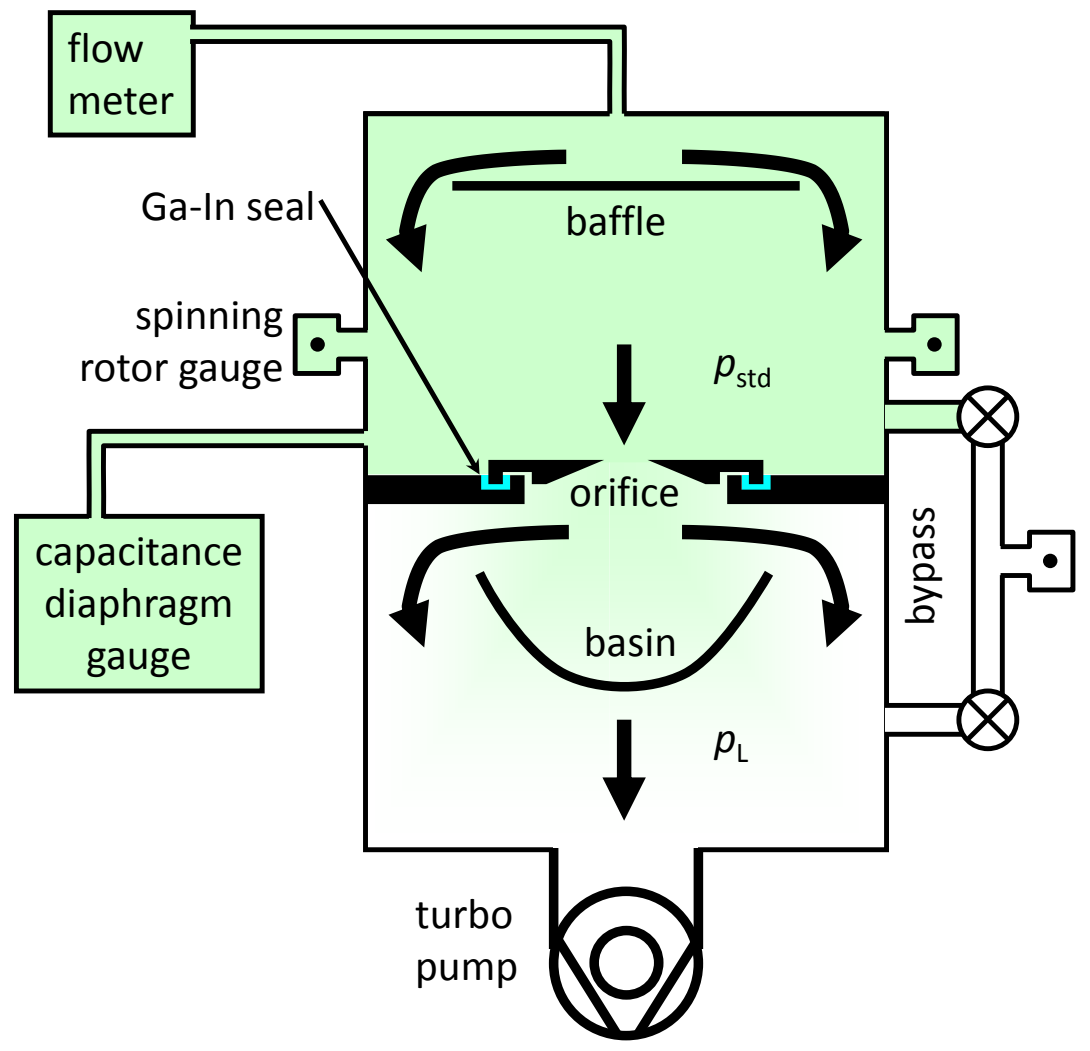

Figure 9. Simplified schematic of the vacuum chamber (not to scale). Gas flows from the flow meter to the upper chamber, through the orifice, and through the lower chamber to the turbo pump. 


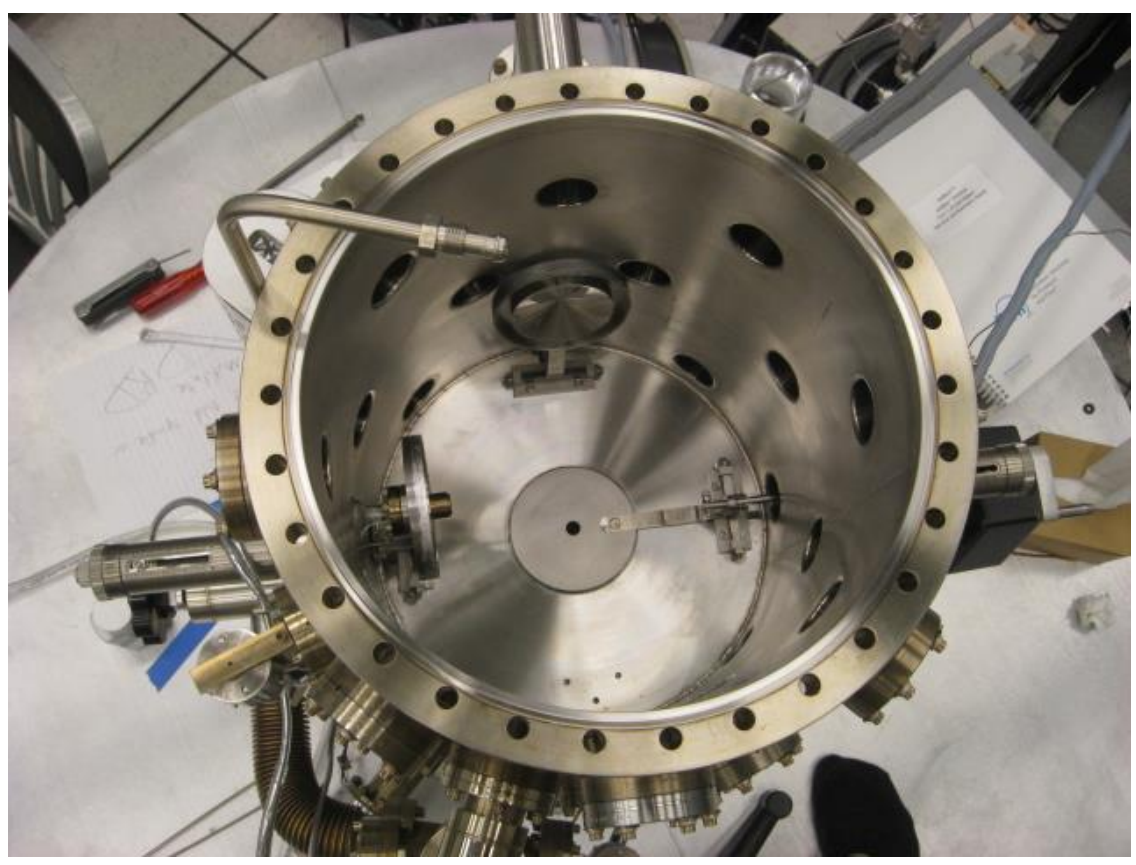

Figure 10. View of the upper half of the vacuum chamber. The $11 \mathrm{~mm}$ orifice plate is in place.

Table 2. The number and function of the (2.75 inch CF or DN40) flanges located on the top of the vacuum chamber and on the sides of the upper and lower halves of the chamber. SRGs to be calibrated are attached to the blank flanges on the upper chamber.

\begin{tabular}{lccc}
\multicolumn{1}{c}{ flange function } & top & upper & lower \\
\hline gas input & 1 & & 1 \\
blank & 1 & 14 & 1 \\
observation window & 1 & 1 & \\
SRG check standard & 3 & \\
crank to lift orifice & 3 & \\
CDG package & 1 & \\
ionization gauge & 2 & 2 \\
upper-lower bypass & 1 & 1 \\
mass spectrometer & & 1 \\
\hline total & $\mathbf{4}$ & $\mathbf{6}$ \\
\hline *One check standard is mounted on the bypass pumping \\
line and can view either the upper or lower chamber.
\end{tabular}

In the upper chamber, a horizontal circular plate located $2 \mathrm{~cm}$ below the top flange acts as a baffle and ensures that a gas molecule entering through the top of the chamber will collide several times with the chamber wall before it enters a gauge or passes through the orifice. The collisions randomize the molecular velocities, a condition that is necessary for pressure uniformity and is assumed in the conductance calculation. 
At the bottom of the upper chamber, the orifice is mounted in the horizontal plate that separates the two halves of the chamber. The mounting scheme allows the orifice to be changed without opening the vacuum chamber. It comprises a large central hole in the plate, a groove that surrounds the large hole, a stainless steel disk that covers the hole, and a crank attached to the disk. The orifice is located in the middle of the disk, which is called the office plate. In normal operation a lip on the orifice plate's lower surface fits into the groove, which is filled with a GaIn eutectic alloy. The alloy is liquid and has a negligible vapor pressure at room temperature and provides a tight seal between the orifice-plate and horizontal plate. Thus, the orifice is the only path between the upper and lower chambers.

Turning the crank handle on the outside of the chamber lifts the orifice plate and opens the central hole. The hole can then be covered by one of the other two disks that contain an orifice. The $11 \mathrm{~mm}$ orifice is used for a typical calibration at $20 \mathrm{mPa}$, while the $2 \mathrm{~mm}$ orifice allows pressures above $100 \mathrm{mPa}$. Table 4 in a later section gives the conductances of these two orifices; the third disk contains an experimental conductance made from a microchannel plate.

As discussed in [2], the inlet and outlet flows perturb the pressure uniformity in the upper chamber. The baffle at the top of the chamber reduces the inlet perturbation. The small area of the orifice, relative to the surface area of the chamber, reduces the outlet perturbation; the resulting error in a similar chamber with an $11 \mathrm{~mm}$ orifice was estimated to be only $0.04 \%$ [2].

The lower chamber contains a stainless steel basin that catches any liquid metal that might fall from the groove into the lower chamber, thereby preventing damage to the turbo pump. The basin also acts as a baffle so that the pressure immediately below the orifice is similar to that read by an SRG check standard that is connected to the side of the lower chamber. That SRG is used to measure the pressure ratio $R_{P}$. At the bottom of the lower chamber is a gate valve that opens to the turbo pump.

The turbo pump has a nominal pumping speed of $620 \mathrm{~L} / \mathrm{s}$, which is sufficient to evacuate the chamber to a low base pressure at zero flow and to create a large pressure ratio across the orifice at nonzero flow. It is backed by either a dry-sealed scroll pump or an oil-sealed rotary pump in series with a zeolite adsorbate trap. Vibration of the pumps is a negligible contribution to the SRG noise.

Part of the external plumbing is a bypass duct that is connects the upper and lower halves and includes an SRG check standard and two all-metal valves. Opening only the upper valve connects the SRG to the upper chamber, and opening only the lower valve connects it to the lower chamber. Opening both valves speeds the pumping of the upper chamber. Another part of the external plumbing (not shown in Figure 9) is tubing that, depending on the valve position, connects the flow meter to either the top of the upper chamber or the side of the lower chamber. Flow directed into the lower chamber allows lower pressures to be achieved in the upper chamber than would be achieved by the same flow directed to the upper chamber, but this feature of the standard is not relevant for SRG calibrations.

An ITS-90 calibrated platinum resistance thermometer is used to measure the temperature of the outer wall of the vacuum chamber. 


\subsection{The flow meter}

\subsubsection{Principle of operation}

Two constant-pressure flow meters are used to generate the gas flows needed to calibrate vacuum gauges and vacuum leak artifacts. Both the "piston flow meter" (PFM) and the "bellows flow meter" (BFM) generate a known flow by allowing gas to leak from a small variable volume, through an impedance, and into the vacuum chamber. As the gas leaks out, the pressure in the volume is held constant by slowly inserting a piston into the volume. Assuming the ideal gas law and combining the gas pressure $p$ and temperature $T$ with the known rate of change of the volume $\dot{V}$ yields the molar flow rate as

$$
\dot{n}=-\frac{p \dot{V}}{R T}+\dot{n}_{\text {outgas }} .
$$

Here $R$ is the gas constant, and $\dot{n}_{\text {outgas }}$ is molar flow due to outgassing from the interior walls of the flow meter. (The variables $p$ and $T$ in this subsection refer to the flow meter and not to the vacuum chamber.) The design of the flow meter is described in an article by McCulloh et al. [25], and the outgassing correction of Eq. (8) is derived in an internal report [7].

\subsubsection{Uncertainty}

The uncertainties of the piston and bellows flow meters are described in an internal report [8]. The standard relative uncertainty of the flow rate is

$$
u_{\dot{n}} \square\left[\left(u_{p}^{2}+u_{\Delta V}^{2}+u_{T}^{2}+u_{\Delta t}^{2}\right)+k_{N}\left(\frac{V_{0}}{\Delta V}-1\right)^{2}\left(u_{\delta p}^{2}+u_{\delta T}^{2}\right)+\left(\frac{\dot{n}_{\text {outgas }}}{\dot{n}}\right)^{2} u_{\text {outgas }}^{2}+u_{\text {misc }}^{2}\right]^{1 / 2},
$$

where

$$
\begin{aligned}
p & =\text { fill pressure } \\
T & =\text { temperature } \\
k_{N} & =\text { statistical factor }\left(k_{N}=0.546 \text { for } N=18\right) \\
V_{0} & =\text { initial volume at start of piston stroke } \\
\Delta V & =\text { volume of stroke } \\
\Delta t & =\text { duration of stroke } \\
\delta P & =\text { pressure drift during stroke } \\
\delta T & =\text { temperature drift during stroke }
\end{aligned}
$$

In Eq. (9), all squared factors are dimensionless. For each of the variables $p, T, V_{0}, \Delta V, \Delta t$, and $\dot{n}_{\text {outgas }}$, the relative uncertainty $u_{\mathrm{X}}$ of variable $X$ is calculated by dividing its absolute uncertainty $U_{\mathrm{X}}$ by the value of the variable. $\left(u_{\mathrm{p}}=U_{\mathrm{p}} / p\right.$, etc.) For the drifts $\delta p$ and $\delta T$, the absolute uncertainty is normalized by the associated variable, namely $u_{\delta p}=U_{\delta p} / p$ and $u_{\delta T}=U_{\delta T} / T$. The statistical factor $k_{N}$ accounts for using $N$ measurements of displacement during the piston stroke instead of only the end points $(N=2)$. Eq. (9) is valid even when $\dot{n}$ is very small and comparable to $\dot{n}_{\text {outgas }}$ because the uncertainty will then be dominated by the uncertainty of $\dot{n}_{\text {outgas }}$.

To estimate the fill pressure required for a given flow rate an approximate relation can be used: 


$$
p \square \frac{\dot{n} R T \Delta t}{\Delta V} .
$$

Here $\Delta t=1000 \mathrm{~s}$ is the typical duration of a run, and the volume displaced by the piston is $\Delta V=12.9 \mathrm{~cm}^{3}$ for the "inch" piston and $2.0 \mathrm{~cm}^{3}$ for the "cm" piston. In other words, the ratio between the fill pressure and the flow rate is fixed independent of the flow rate. In practice $\Delta t$ is a constant only in free molecular flow. At a $\mathrm{N}_{2}$ fill pressure of $100 \mathrm{kPa}$, for example, $\Delta t$ can decrease from the molecular flow value by roughly a factor of 2 in the BFM.

Table 3 lists values, calculated from Eq. (9), of the Type B uncertainty contributions at two flow rates for each of the flow meters [8]. A flow rate of $1 \times 10^{-7} \mathrm{~mol} / \mathrm{s}$ will produce a pressure of approximately $23 \mathrm{mPa}$ in the upper chamber of the mid-range chamber. This is a typical calibration pressure. Either flow meter can produce the flow, but a flow of $1 \times 10^{-7} \mathrm{~mol} / \mathrm{s}$ is close to the upper flow limit that can be produced in the BFM using a $133 \mathrm{kPa}$ Yokogawa resonance silicon gauge (RSG) to measure the BFM fill pressure. At a flow of $1 \times 10^{-7} \mathrm{~mol} / \mathrm{s}$, we see from Table 3 that the PFM uncertainty is $0.2 \%(k=2)$ and the PFM uncertainty is approximately $0.3 \%(k=2)$. The uncertainty is calculated at the time of the calibration and can vary from these values by a small amount. For example, the duration of the run is often decreased (or increased) from the values used in Table 3 , and the long-term stability of the pressure gauge, included in $u_{p}$, is occasionally updated to reflect the history of the gauge. The Type A uncertainty is also calculated from the repeatability of each gauge's reading, but this contribution to the total uncertainty is usually negligible. The fill pressures, turn times, pressure uncertainties, and outgassing rates used for the uncertainty estimates in Table 3 were taken from 2011 PFM data and 2014 BFM data. For upper chamber pressures greater than $23 \mathrm{mPa}$, the PFM is typically used and the uncertainty varies little with flow for $\dot{n}>1 \times 10^{-7}$.

Table 3. Contributions to the relative flow Type B uncertainty for the piston flow meter (PFM) and the bellows flow meter (BFM) at various molar flow rates. All values are relative standard uncertainties expressed in percent. The diameter of the piston used in the uncertainty determination is shown in parenthesis.

\begin{tabular}{rc|cc|cc}
\hline \multicolumn{1}{r|}{ contribution } & \multicolumn{2}{c|}{ PFM (2.54 cm piston) } & \multicolumn{2}{c}{ BFM (1 cm piston) } \\
& & $10^{-7} \mathrm{~mol} / \mathrm{s}$ & $\begin{array}{c}5 \times 10^{-8} \\
\mathrm{~mol} / \mathrm{s}\end{array}$ & $10^{-7} \mathrm{~mol} / \mathrm{s}$ & $10^{-11} \mathrm{~mol} / \mathrm{s}$ \\
piston area & $u_{A}$ & 0.001 & 0.001 & 0.001 & 0.001 \\
time & $u_{L}$ & 0.100 & 0.100 & 0.100 & 0.100 \\
micrometer stroke & $u_{\Delta t}$ & 0.001 & 0.001 & 0.001 & 0.001 \\
\hline volume rate of change & & $\mathbf{0 . 1 0 0}$ & $\mathbf{0 . 1 0 0}$ & $\mathbf{0 . 1 0 0}$ & $\mathbf{0 . 1 0 0}$ \\
temperature calibration & $u_{T . \mathrm{PRT}}$ & 0.010 & 0.010 & 0.010 & 0.010 \\
temperature random & $u_{T . \text { random }}$ & 0.002 & 0.002 & 0.002 & 0.002 \\
temperature drift & $k_{18}\left(V_{0} / \Delta V-1\right) u \delta T$ & 0.002 & 0.002 & 0.014 & 0.014 \\
\hline temperature & & $\mathbf{0 . 0 1 0}$ & $\mathbf{0 . 0 1 0}$ & $\mathbf{0 . 0 1 7}$ & $\mathbf{0 . 0 1 7}$ \\
pressure gauge & $u_{P}$ & 0.014 & 0.066 & 0.101 & 0.313 \\
pressure drift & $k_{18}\left(V_{0} / \Delta V-1\right) u_{\delta P}$ & 0.004 & 0.004 & 0.000 & 0.082 \\
\hline
\end{tabular}




\begin{tabular}{rr|cc|cc}
\hline pressure & & $\mathbf{0 . 0 1 7}$ & $\mathbf{0 . 0 6 6}$ & $\mathbf{0 . 1 0 1}$ & $\mathbf{0 . 3 2 3}$ \\
\hline outgassing & $\boldsymbol{u}_{\text {outgas }}$ & $\mathbf{0 . 0 0 1}$ & $\mathbf{0 . 0 0 1}$ & $\mathbf{0 . 0 0 0}$ & $\mathbf{0 . 1 5 5}$ \\
& & & & \\
leak past sliding seal & $u_{\text {leak }}$ & 0.010 & 0.010 & -- & 0.000 \\
gas purity & $u_{\text {purity }}$ & 0.010 & 0.010 & 0.010 & 0.010 \\
\hline miscellaneous & $\boldsymbol{u}_{\text {misc }}$ & $\mathbf{0 . 0 1 4}$ & $\mathbf{0 . 0 1 4}$ & $\mathbf{0 . 0 1 0}$ & $\mathbf{0 . 0 1 0}$ \\
\hline total flow uncertainty & $\boldsymbol{u}$ & $\mathbf{0 . 1 0 2}$ & $\mathbf{0 . 1 2 1}$ & $\mathbf{0 . 1 4 3}$ & $\mathbf{0 . 3 7 3}$ \\
\hline
\end{tabular}

\subsection{The orifice}

\subsubsection{Construction}

Each orifice was designed so that its conductance could be easily calculated. An ideal orifice would be a circular hole in a plate of zero thickness, while the actual orifice was made by grinding a sphere into a plate with a conical depression [1], as indicated in Figure 11.

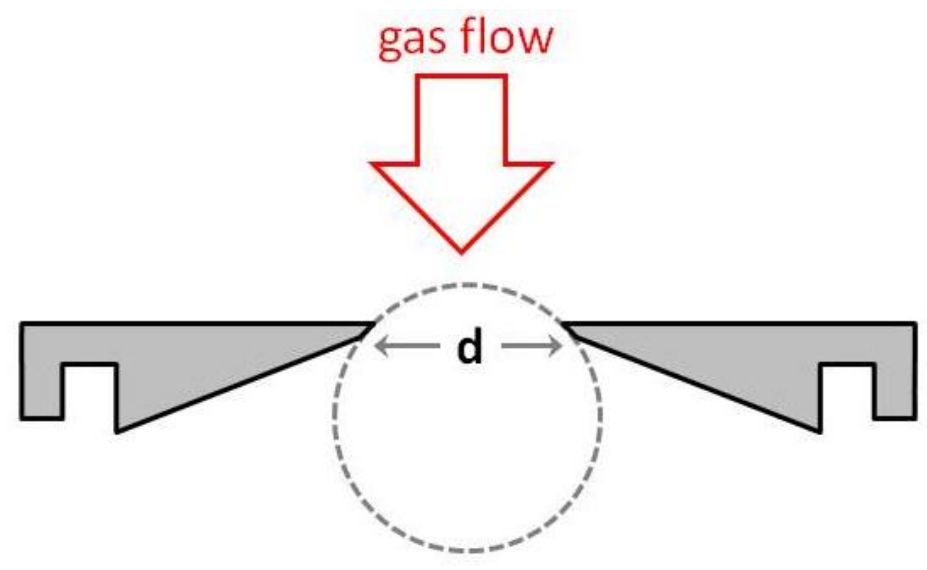

Figure 11. Cross section of the orifice, which was made by grinding a sphere into a plate with a conical depression. The cone angle and the relative sizes of the orifice diameter $d$ and the sphere diameter shown here are similar to those of the actual orifices. The lip that surrounds the plate fits into a circular groove filled with liquid Ga-In.

\subsubsection{Conductance at low pressure}

The conductance for molecular flow through the orifice is

$$
C_{0}=\frac{K A}{4} \bar{v}=K\left(\frac{\pi d^{2}}{16}\right)\left(\frac{8 R T}{\pi M}\right)^{1 / 2},
$$

where 


$$
\begin{aligned}
K & =\text { Clausing factor } \\
A & =\text { orifice area } \\
\bar{v} & =\text { average molecular speed } \\
d & =\text { orifice diameter } \\
R & =\text { gas constant } \\
T & =\text { temperature } \\
M & =\text { molecular weight }
\end{aligned}
$$

The Clausing factor, $K<1$, is the transmission probability for a molecule to pass through the orifice and not bounce back due to a collision with the side of the orifice. Looney [5] calculated the value of $K$ from a series model based on the orifice dimensions measured at NIST [26]. Table 4 gives the values of $d, K$, and $C_{0}$ for both orifices.

Table 4. Molecular flow characteristics of the two orifices and their standard uncertainties; $P_{K n=1}$ is the pressure at which the Knudsen number is 1 . The characteristics were calculated for nitrogen gas at $298.15 \mathrm{~K}$.

\begin{tabular}{rc|cc|cc|c}
\multicolumn{1}{c}{$\mathbf{2 ~ \mathbf { ~ m m }} \mathbf{1 1} \mathbf{~ m m}$} \\
\hline diameter & $d$ & $2.218 \pm 0.002$ & {$[26]$} & $11.158 \pm 0.005$ & {$[26]$} & $\mathrm{mm}$ \\
Clausing factor & $K$ & $0.9857 \pm 0.0003$ & {$[5]$} & $0.9845 \pm 0.0003$ & {$[5]$} & $\mathrm{L} / \mathrm{s}$ \\
conductance & $C_{0}$ & $0.4520 \pm 0.0007$ & {$[5]$} & $11.423 \pm 0.010$ & {$[5]$} & $\mathrm{L} / \mathrm{s}$ \\
transition flow limit & $p_{\mathrm{Kn}=1}$ & 6.74 & & 1.34 & & $\mathrm{~Pa}$ \\
\hline
\end{tabular}

\subsubsection{Conductance at high pressure}

At large pressures, the flow through the orifice is no longer molecular, and the conductance increases with pressure as a function of the Knudsen number $K n$. For a pure gas,

$$
C(p)=C_{0}[1+f(K n(p))] \cong C_{0}\left[1+\alpha_{1} p\right] .
$$

Here $C_{0}$ is the orifice conductance in molecular flow, and $K n$ is defined as the ratio of the mean free path $\lambda$ to the orifice radius $d / 2$,

$$
K n \equiv \frac{\lambda}{d / 2}=\frac{2}{d}\left(\frac{2 R T}{M}\right)^{1 / 2} \frac{\eta}{p},
$$

where $\eta$ is the gas viscosity. Hyland [4] measured the value of the pressure coefficient $\alpha_{1}$ for both orifices by using seven SRGs at pressures up to $0.22 \mathrm{~Pa}$, and Fedchak [10] measured $\alpha_{1}$ for the $11 \mathrm{~mm}$ orifice by using a CDG as well as seven SRGs up to the higher pressure of $0.52 \mathrm{~Pa}$. The results are listed in Table 5. For the $2 \mathrm{~mm}$ orifice, the best correction to the conductance for transitional flow is obtained by a polynomial fit:

$$
C(p) \square C_{0} \sqrt{\frac{T}{295.15 \mathrm{~K}}}\left(1+C_{1} \sqrt{\frac{T}{295.15 \mathrm{~K}}} p+C_{2} \sqrt{\frac{T}{295.15 \mathrm{~K}}} p^{2}\right),
$$

These coefficients were empirically determined in 2000 [27] over a pressure range of $0.5 \mathrm{~Pa}$ to $4 \mathrm{~Pa}$ and are given in Table 6 .

Also listed in Table 5 is the result of a DSMC (direct simulation Monte Carlo) calculation by Sharipov [28] for near-molecular-flow through a thin orifice. His numerical results for a hard- 
sphere potential and pressures in the range $K n^{-1}<2$ (corresponding to $P<2.7 \mathrm{~Pa}$ nitrogen through the $11 \mathrm{~mm}$ orifice) can be summarized as

$$
\alpha_{1}=\left[\frac{C(P)}{C_{0}}-1\right] \frac{1}{P}=\left[\frac{A_{U}}{K n(P)}+\frac{A_{L}}{K n\left(P_{L}\right)}\right] \frac{1}{P}=\left[A_{U}+\frac{A_{L}}{R_{P}}\right] \frac{d}{2 \eta}\left(\frac{M}{2 R T}\right)^{1 / 2},
$$

where $A_{\mathrm{U}}=0.13$ and $A_{\mathrm{L}}=0.20$. The calculated value for $\alpha_{1}$ given by Eq. (15) assumes that the pressure ratio $R_{P}$ is independent of $P$. The actual pressure dependence of $R_{P}$, shown in Figure 12, contributes negligible error to the calculated values listed in Table 5.

Table 5. The first-order pressure coefficient $\alpha_{1}\left(\mathrm{~Pa}^{-1}\right)$ and its standard uncertainty for the two orifices at $296.15 \mathrm{~K}$ (the temperature of the 2010 measurements). Sharipov's results are from a DSMC hard-sphere calculation.

\begin{tabular}{rrcccc} 
year & reference & method & gas & $\mathbf{2 ~} \mathbf{~ m m}$ & $\mathbf{1 1} \mathbf{~ m m}$ \\
\hline 1996 & Hyland [4] & SRG & $\mathrm{N}_{2}$ & $0.020 \pm 0.001$ & $0.1153 \pm 0.0024$ \\
1996 & Hyland [4] & SRG & $\mathrm{Ar}$ & & $0.1091 \pm 0.0022$ \\
2004 & Sharipov [28] & DSMC calc & $\mathrm{N}_{2}$ & 0.0206 & 0.104 \\
2004 & Sharipov [28] & DSMC calc & $\mathrm{Ar}$ & 0.0194 & 0.097 \\
2010 & Fedchak [10] & SRG \& CDG & $\mathrm{N}_{2}$ & & $0.1235 \pm 0.0031$ \\
\hline
\end{tabular}

Table 6. Measurements of the polynomial coefficients in Eq. (14) for the $2 \mathrm{~mm}$ orifice at 296.15 K. Two fits are given.

\begin{tabular}{lrccc} 
& $\boldsymbol{C}_{\boldsymbol{0}}(\mathbf{L} / \mathbf{s})$ & $\boldsymbol{C}_{\boldsymbol{1}}\left(\mathbf{P a}^{-1}\right)$ & $\boldsymbol{C}_{\boldsymbol{2}}\left(\mathbf{P a}^{-\mathbf{2}}\right)$ & $\boldsymbol{u}(\boldsymbol{k}=\mathbf{1})$ \\
\hline Second order & 0.451768 & .0242902 & $-8.9971 \times 10^{-4}$ & $0.05 \%$ \\
First order & 0.451768 & .0212082 & & $0.18 \%$ \\
\hline
\end{tabular}

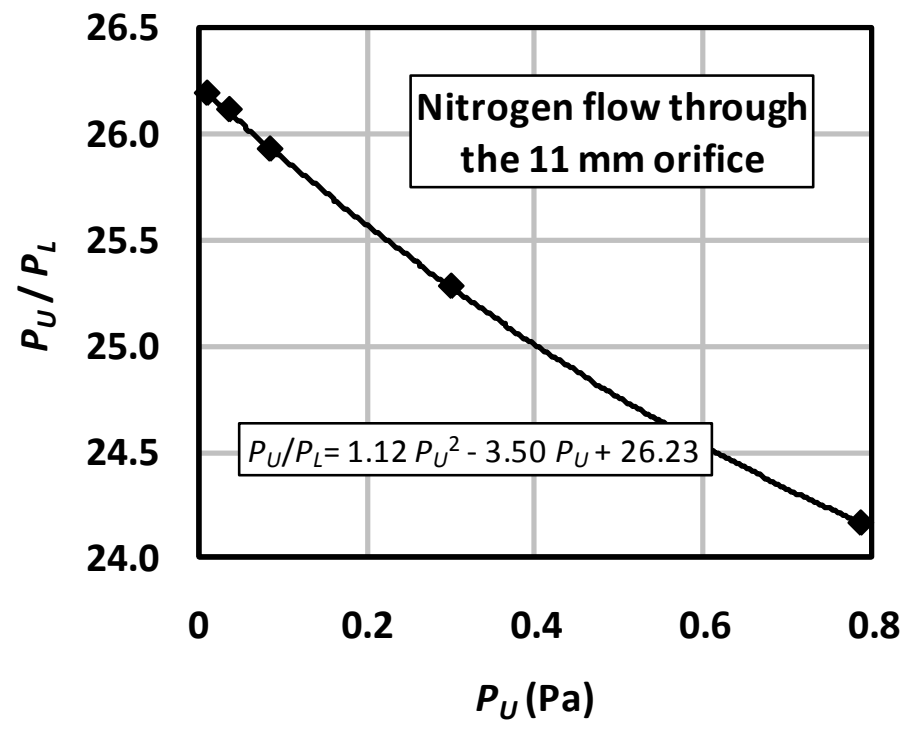

Figure 12. The pressure ratio for nitrogen flow through the $11 \mathrm{~mm}$ orifice [10]. 


\section{Operation of the standard}

The measurement equations for determining the effective accommodation coefficient can be derived by dropping the last term in Eq. (5) and combining it with Eq. (6):

$$
\sigma=\frac{1}{p_{\text {std }}}\left(\frac{2 \pi R T}{M}\right)^{1 / 2} \frac{\rho d}{10}\left(D C R-D C R_{0}\right),
$$

In Eq. (16) $T$ is the gas temperature and it is assumed to be the same as the chamber of the standard. There is no uncertainty associated with $\rho$ and $d$ because their values are the same nominal values that were used to determine the pressure via Eq. (5).

To calculate the standard pressure $p_{s t d}$, substitute $q_{p V}=\dot{n} R T$ and Eq. (12) for $C(p)$ into Eq. (4). The resulting expression depends on the correction term $\alpha_{1} p_{s t d}$, and using the approximation $p_{\text {std }} \cong p_{s t d, 0}$ in that term yields

$$
p_{\text {std }}=\frac{\dot{n} R T}{C_{0}\left(1+\alpha_{1} p_{s t d, 0}\right)}\left(\frac{R_{P}}{R_{P}-1}\right),
$$

The error due to the approximation is negligible in comparison to the uncertainty, as shown in Section 1.9. Values for $C_{0}$ and $\alpha_{1}$ are taken from the NIST measurements given in Table 5. Most NIST calibrations of SRGs are performed near $0.02 \mathrm{~Pa}$. At this pressure, the typical reproducibility of the vacuum decrement contributes only $0.01 \%$ to the standard uncertainty, while the pressure correction for the $11 \mathrm{~mm}$ orifice is less than $0.3 \%$. However, the pressure correction is greater than $1 \%$ for calibrations performed at $0.1 \%$.

\subsection{Calibrations below 0.1 Pa}

The medium range vacuum chamber is exposed to room air only when SRGs are mounted. Unlike the chamber used to calibrate ionization gauges, it is usually not baked because one day of pumping is sufficient to reduce the base pressure to below $1 \times 10^{-5} \mathrm{~Pa}$, which is comparable to the pressure corresponding to the reproducibility of the vacuum decrement.

Preparation for an SRG calibration includes the following.

1. The rotors, controllers, heads, and other associated equipment are unpacked and inspected for damage. Serial numbers and other markings are recorded in a notebook.

2. If the rotor is new and has no calibration history at NIST:

a. Remove each rotor from its thimble, inspect it under an optical microscope for scratches and corrosion, and wash it in ethanol. Swab each thimble with a series of ethanol-wetted cotton swabs until the swab shows no visible discoloring.

b. Weigh each rotor $( \pm 1 \mathrm{mg})$ and measure its diameter $( \pm 1 \mu \mathrm{m})$.

3. Place the rotors into their thimbles, mount the thimbles and suspension heads onto the chamber, and begin evacuating the chamber.

4. Suspend the rotors, spin up the rotors, and measure the rotation signal strengths. Address any electronic problems, such as a failure to suspend the rotor or a weak signal. Remagnetize a problem rotor if necessary. 
5. The rotor must be mounted and suspended at least one day before any reliable data can be recorded. This ensures that the rotor is in temperature equilibrium with the chamber.

6. Record residual drag data for at least one day with the chamber pressure below $1 \times 10^{-5} \mathrm{~Pa}$. Fit the data for $D C R_{0}(f)$ by a linear function of frequency $f$.

7. Start a gas flow sufficient to obtain the desired pressure, typically $0.02 \mathrm{~Pa}$.

Prior to 2014, step 2 was performed on every rotor. NIST's own studies indicate that ethanol rinses and handling the rotors reduces the long-term stability; therefore this practice was abandoned and step 2 is presently only performed on rotors with no calibration history. Step 2 may also be performed on rotors if there is evidence that the rotors or thimbles are not clean and will affect the vacuum quality or performance.

The calibration then proceeds as follows.

1. Initiate a control program(s) to record the chamber temperature, gas flow rate, and the decrements reported by the SRG controllers. The typical duration of a calibration point is 10 to 20 minutes.

2. Use Eqs. (17) to obtain the primary standard pressure $p_{\text {std }}$, and use Eq. (16) to obtain the effective accommodation coefficient. The rotor diameter $d$ and density $\rho$ are taken from the NIST SRG calibration database if the rotor has a calibration history at NIST.

Otherwise, the values determined in step 2 in Section 6.2 or nominal values provided by the customer are used.

3. Repeat steps 1 and 2 at least 5 times.

The last steps are as follows.

1. Measure the vacuum decrement again to verify that no significant change occurred.

2. De-suspend the rotors using the braking feature on the controller, turn off the suspension, and remove the suspension heads and thimbles from the vacuum chamber.

3. Re-pack the SRG's and associated equipment.

4. Produce the calibration report, and place a signed copy with the equipment.

5. Seal the boxes and ship the SRGs back to the customer.

\subsection{Calibrations above $0.1 \mathrm{~Pa}$}

Comsa et al. [29] used a static expansion pressure standard to measure SRG linearity in noble gases at pressures as high as $2 \mathrm{~Pa}$ and found that, for argon, the error was approximately $0.3 \%$ at $0.2 \mathrm{~Pa}$. (Consistent with the dependence of the mean free path on molecular weight, the error was larger for the heavier gases.) At higher pressures, the error is much larger [30].

\subsubsection{Pressures up to $2 \mathrm{~Pa}$}

The procedure here is similar to that at lower pressures except that the $2 \mathrm{~mm}$ orifice may be used. The $2 \mathrm{~mm}$ orifice has a conductance which is 25 times smaller than the $11 \mathrm{~mm}$ orifice. For a given gas flow $\dot{n}$ into the upper chamber, $p_{\text {std }}$ will be 25 times larger when the $2 \mathrm{~mm}$ orifice is used as compared to when the $11 \mathrm{~mm}$ orifice is used. Therefore, to produce the same pressure $p_{s t d}$ with the $2 \mathrm{~mm}$ and $11 \mathrm{~mm}$ orifices, the $2 \mathrm{~mm}$ orifice requires a gas flow that is 25 times smaller. Since the BFM is limited to about $10^{-7} \mathrm{~mol} / \mathrm{s}$ and the PFM is limited to about $10^{-6} \mathrm{~mol} / \mathrm{s}$, using the $2 \mathrm{~mm}$ orifice becomes necessary at the higher pressures. Figure 13 shows measurements made with nitrogen flow through both orifices and demonstrates the consistency 
obtained with the medium range pressure standard. Measurements made using the two orifices agree to within the measurement uncertainty. The values acquired with the $11 \mathrm{~mm}$ orifice differ by only $0.07 \%$, which is consistent with the Type A uncertainty (see the discussion in the next section), and the typical slope observed for the accommodation coefficient is in the range of 0.1 $\mathrm{Pa}$ to $2 \mathrm{~Pa}$ (see discussion below). The values acquired with the $2 \mathrm{~mm}$ orifice agree to within $0.02 \%$ up to $0.15 \mathrm{~Pa}$ and also are consistent with the Type A uncertainty. Between 0.1 and 0.2 $\mathrm{Pa}$, any pressure dependence of the accommodation coefficient is too small to be observed.

The rotor-thimble "viscous" correction is significant above $0.2 \mathrm{~Pa}$ because the mean free path is comparable to or smaller than the diameter of the thimble. The resulting viscous flow around the rotor reduces the coupling between the rotor and the thimble, and using Eq. (5) without the viscous correction will yield a value for $p_{S R G}$ that is too small even after applying the pressure correction to the orifice conductance. Figure 14 shows the accommodation coefficient for 9 rotors for pressures up to $1 \mathrm{~Pa}$. The accommodation coefficient was determined using Eq. (7). A linear fit was applied to the accommodation coefficient for pressures between $0.1 \mathrm{~Pa}$ and $1.0 \mathrm{~Pa}$; these are shown as lines in Figure 14. The slopes for all but one rotor were about $0.015 \mathrm{~Pa}^{-1}$. The linear model of the accommodation coefficient is a good approximation for pressures in the range of $0.1 \mathrm{~Pa}$ to $2 \mathrm{~Pa}$ [19]. The nonzero slope requires that, for calibrations in the range of $0.1 \mathrm{~Pa}$ to $2 \mathrm{~Pa}$, the accommodation coefficient must be measured at a minimum of two pressures.

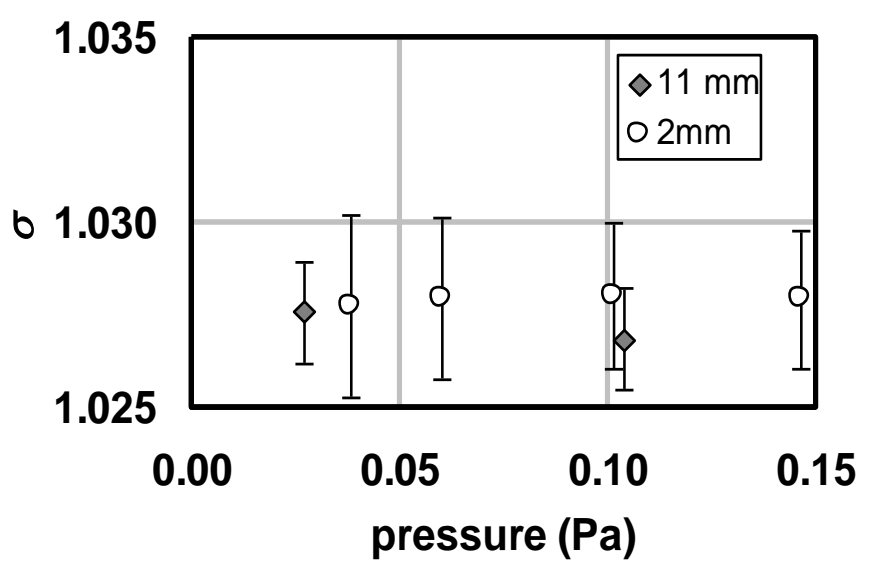

Figure 13. Results for the accommodation coefficient $\sigma$ of a customer rotor in nitrogen. The $11 \mathrm{~mm}$ orifice was used to obtain the value of $\sigma$ at $0.104 \mathrm{~Pa}$ as well as at the standard pressure of $0.027 \mathrm{~Pa}$. The $2 \mathrm{~mm}$ orifice was used to obtain the values at other pressures. The error bars indicate the standard uncertainty $(k=1)$ for each measurement. The uncertainties are essentially Type $B$ because the Type A components are negligible. 


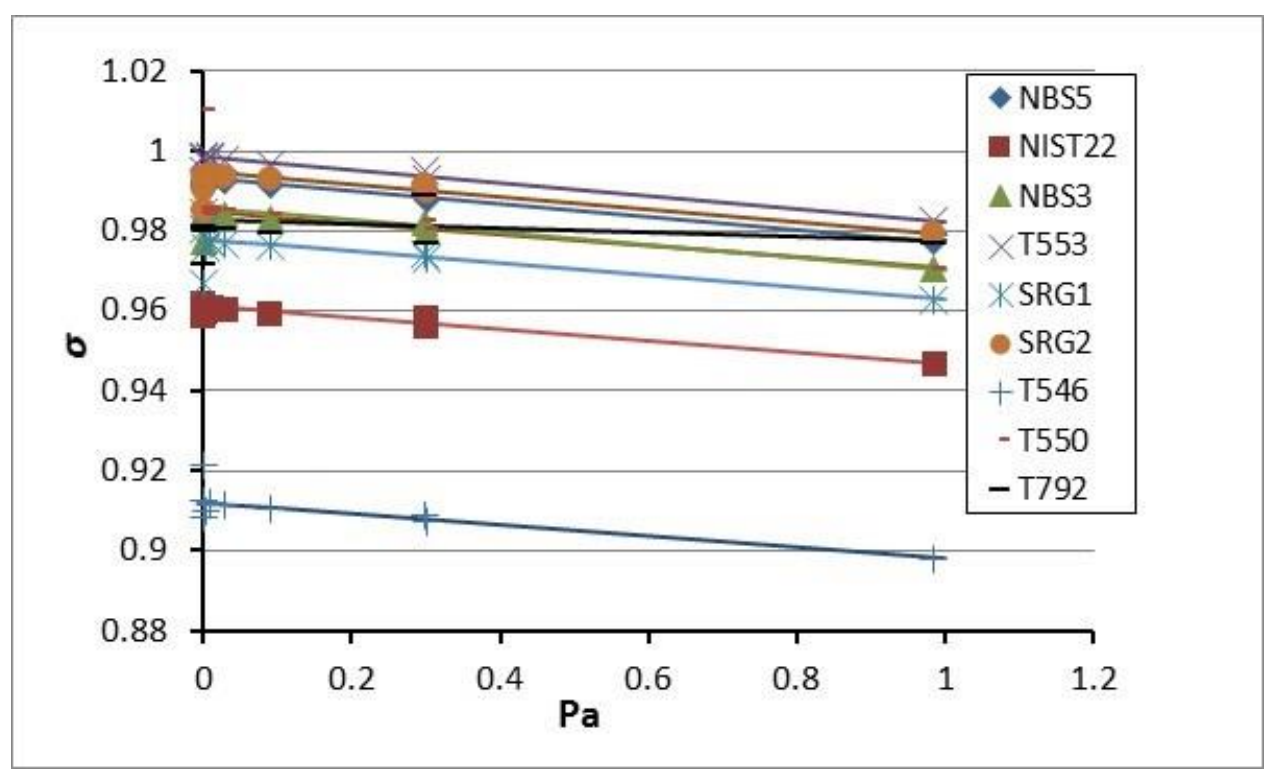

Figure 14. The accommodation coefficient $\sigma$ as a function of pressure for 9 rotors. The black lines represent linear fits to the data for $p>0.1$ Pa. The slopes for all of the rotors except $\mathrm{T} 792$ were approximately $0.015 \mathrm{~Pa}^{-1}$.

\subsubsection{Pressures above $2 \mathrm{~Pa}$}

The mid-range pressure standard can generate known pressures up to $4 \mathrm{~Pa}$ by using the $2 \mathrm{~mm}$ orifice. The main limitations to using the standard at higher pressures are the maximum flow rate of the PFM and insufficient knowledge of the orifice conductance above $4 \mathrm{~Pa}$. At higher pressures, the SRGs are calibrated by closing off the vacuum pump, filling the chamber to the calibration pressure, and using one or more calibrated capacitance diaphragm gauges (CDG) as a pressure reference. The CDGs are calibrated against the NIST liquid-column manometers; the lowest range $\mathrm{CDG}$ in use (133 Pa full scale) has a relative standard uncertainty of approximately $0.5 \%$ at $1 \mathrm{~Pa}$. In general, we do not recommend using SRGs as transfer standards above about 1 $\mathrm{Pa}$.

As previously pointed out, the rapid deceleration of the rotor at high pressures causes eddy current heating that leads to temperature instability of the rotor and adds uncertainty that is difficult to quantify. Nevertheless, rotors can be calibrated above $2 \mathrm{~Pa}$ by using the two-point calibration scheme described by Šetina [31] Šetina's scheme is necessary as the linear model described in the previous section is not valid above $2 \mathrm{~Pa}$. We will now give a brief outline of the calibration technique of Šetina, and the reader should refer to Ref. [31] for additional details and definitions.

In the transition regime, the rotor deceleration rate can be expressed as:

$$
D C R=\frac{5 \eta^{*}}{\rho d \delta}
$$

As usual, $\rho$ is the rotor density and $d$ is the rotor diameter. Also, $\delta$ is an unknown "Knudsen length" that characterizes the gap between the rotor and the thimble, and $\eta^{*}$ is the effective viscosity which is related to the true viscosity $\eta$ by: 


$$
\eta^{*}=\eta\left[1+\left(\frac{2 K n}{\sigma}\right)\left(A_{1}+\left(1-A_{1}\right) \exp \left(-\frac{A_{2}}{K n}\right)\right)\right]^{-1},
$$

Here $A_{1}$ and $A_{2}$ are empirical constants, and the Knudsen number $K n$ is defined by

$$
K n \equiv \frac{\lambda}{\delta}=\frac{2 \eta}{\delta p}\left(\frac{\pi R T}{8 M}\right)^{1 / 2}
$$

Note that this is a different definition of $K n$ than given in Eq. (13), but is re-defined here to be consistent with Ref. [31]. Eqs. (18)-(20) can be combined to give the decrement, DCR, as a function of pressure $p$. The decrement is thus seen to depend on the effective accommodation coefficient $\sigma$ determined in the free molecular flow regime and the parameters $\delta, A_{1}$, and $A_{2}$. Šetina found that the parameters $A_{1}$ and $A_{2}$ depend on the rotor-thimble geometry, but nominal values can be used for a given rotor diameter (assuming all rotor thimbles are the same size). For example, for $d=4.5 \mathrm{~mm}$ we have $A_{1}=0.67945$ and $A_{2}=0.878$, and for $d=4.76 \mathrm{~mm}$ we have $A_{1}=00.69143$ and $A_{2}=0.0 .881$. Thus, using Eqs. (18)-(20), calibrating a rotor only requires 2 calibration pressures: one pressure in the molecular flow regime to determine $\sigma$, and one in the viscous regime to determine $\delta$. Using the above values for $A_{1}$ and $A_{2}$, Šetina determined $\delta$ near $100 \mathrm{~Pa}$ and found that model given by Eqs. (18)-(20) resulted in errors less than $2 \%$ at pressures up to $130 \mathrm{~Pa}$.

\section{Analysis and uncertainty}

Three quantities are calculated for each calibration point: the primary standard pressure $p_{\text {std }}$ from Eq. (17), the apparent SRG pressure $P_{\mathrm{SRG}}$ from Eq. (5), and their ratio $\sigma=p_{\mathrm{SRG}} / p_{\text {std }}$. The calculation of $p_{\text {SRG }}$ uses assigned values for the rotor's density and diameter. Usually the values are those measured at the time of the rotor's first calibration, although nominal values are used in some cases. The accuracy of the values of density and diameter are unimportant for the calibration as long as consistent values are used.

The following subsection discusses the uncertainty of the newly calibrated SRG, which is dominated by the uncertainties of flow meter (Table 3 ) and the orifice conductance (Table 4). The next subsection discusses the uncertainty for the user of the calibrated SRG.

\subsection{Uncertainty immediately after calibration}

Since the Standard Calibration Service is for a single pressure less than $0.1 \mathrm{~Pa}$, the following uncertainty analysis applies only to pressures less than $0.1 \mathrm{~Pa}$. To identify the contributions to the uncertainty of $\sigma$, combine Eqs. (4), (5), and (12) to obtain

$$
\sigma=\frac{p_{\mathrm{SRG}}}{p_{\text {std }}}=K_{\sigma} \frac{C_{0}\left(1+\alpha_{1} p_{s t d, 0}\right)}{\dot{n}}\left(1-R_{P}^{-1}\right)\left(D C R-D C R_{0}\right) T^{-1 / 2},
$$

where 


$$
\begin{aligned}
K_{\sigma} & =\text { fixed constant with no uncertainty } \\
\dot{n} & =\text { molar flow rate through chamber } \\
C_{0} & =\text { orifice conductance for molecular flow } \\
\alpha_{1} & =\text { pressure coefficient for orifice conductance } \\
R_{P} & =\text { chamber pressure ratio } \\
D C R & =\text { SRG decrement } \\
D C R_{0} & =\text { SRG vacuum decrement } \\
T & =\text { chamber temperature }
\end{aligned}
$$

The relative standard uncertainty of $\sigma$ implied by Eq. (21) is

$$
u_{\sigma}=\left[u_{\dot{n}}^{2}+u_{C_{0}}^{2}+\left(\alpha_{1} p_{s t d, 0}\right)^{2} u_{\alpha_{1}}^{2}+\frac{u_{R_{P}}^{2}}{R_{P}^{2}}+\left(\frac{D C R_{0}}{D C R-D C R_{0}}\right)^{2} u_{D C R_{0}}^{2}+\frac{u_{T}^{2}}{2^{2}}+u_{\mathrm{A}}^{2}\right]^{1 / 2},
$$

where $u_{X}$ denotes the relative standard uncertainty of quantity $X$. The uncertainty component associated with $p_{s t d, 0}$ is given by $\alpha_{1} p_{\text {stt }, 0} u_{p_{s t d, 0}}$; since $\alpha_{1} \approx 0.1 \mathrm{~Pa}^{-1}, p_{s t d}<0.1 \mathrm{~Pa}$, and $u_{p_{s t d, 0}}<1 \%$, $\alpha_{1} p_{s t d, 0} u_{p_{s d, 0}}<0.01 \%$ this component was not included in Eq. (22). The uncertainty component $u_{\mathrm{A}}$ is a Type A uncertainty that includes contributions from $u_{D C R}$ and other factors which affect the reproducibility. All of the other components are Type B. Table 7 gives a typical uncertainty budget for a calibration at $0.020 \mathrm{~Pa}$. Below we offer additional details of each component. Unless otherwise stated, all components can be taken as $k=1$.

$u_{A}$ : The Type A uncertainty is the reproducibility of the measurement and is determined using statistical methods during the calibration. The accommodation coefficient is typically measured 10 to 20 times during a calibration, and each measurement is typically the mean of 10 to 20 decrement readings. The reproducibility or standard deviation is typically in the range of $0.01 \%$ to $1 \%$, and a typical value can be taken as $0.05 \% . u_{A}$ is calculated from the standard deviation of the mean; $u_{A}=0.02 \%$ is a typical value.

$u_{n}$ : The uncertainty of the gas flow is discussed in Section 5.2.2. For an upper chamber pressure of $0.02 \mathrm{~Pa}, u_{\dot{n}}=0.102 \%$ for the PFM and $0.143 \%$ for the BFM. We use the PFM values for Table 5 .

$u_{C_{0}}$ : The conductance calculation is discussed in Section 5.3.2. $u_{C_{0}}=0.09 \%$ for the $11 \mathrm{~mm}$ orifice.

$u_{\alpha_{1}}$ : A discussion of the uncertainty in $\alpha_{1}$ can be found in Fedchak [10]. With $u_{\alpha_{1}}=2.5 \%$, $\alpha_{l}=0.12 \mathrm{~Pa}^{-1}$, and $p_{\text {std }}=0.02 \mathrm{~Pa}$, the total contribution of $\alpha_{l}$ to the uncertainty is $\alpha_{1} p_{s t d, 0} u_{\alpha_{1}}=0.01 \%$. Thus the uncertainty contribution of $u_{\alpha_{1}}$ is negligible at $20 \mathrm{mPa}$; however, this term is proportional to pressure and cannot be neglected at higher pressures. 
$u_{R_{P}}$ : From Fedchak [9], the uncertainty in the pressure ratio is $u_{R_{P}}=0.37 \%$ and $R_{p} \sim 26.2$, therefore $u_{R_{P}} / R_{P}=0.014 \%$.

$u_{D C R_{0}}$ : Since the residual drag can strongly vary, it is better to estimate the absolute uncertainty contribution of residual rather than the relative uncertainty. The uncertainty contribution due to the residual drag can be re-written as:

$$
\left(\frac{D C R_{0}}{D C R-D C R_{0}}\right) u_{D C R_{0}}=\frac{\Delta D C R_{0}}{D C R-D C R_{0}}=\frac{\Delta p_{S R G, 0}}{p_{S R G}},
$$

Here, $\triangle D C R_{0}$ is the standard uncertainty associated with the residual drag, which is calculated from the standard deviation of several measurements, and $\Delta p_{S R G, 0}$ is the pressure equivalent to $\triangle D C R_{0}$. At $p_{S R G}=20 \mathrm{mPa}, D C R-D C R_{0}$ is approximately $7.5 \times 10^{-6} \mathrm{~s}^{-1}$, while $\triangle D C R_{0}$ is typically around $6 \times 10^{-10}$, but can range from $2 \times 10^{-10}$ to $2 \times 10^{-9}$. For a conservative estimate, we use $\triangle D C R_{0}=$ $2 \times 10^{-9}$, and therefore $\frac{\Delta p_{S R G, 0}}{p_{S R G}}=0.03 \%$ at $20 \mathrm{mPa}$.

Table 7. Relative standard $(k=1)$ uncertainties $u x$, and weighted contributions for the uncertainty components identified in Eq. (22). The sensitivity coefficients, or weights, are also given, except for the residual drag contribution. As explained in Section 7.1, this total contribution was computed without separately computing the weights. These values are typical for a calibration with the $11 \mathrm{~mm}$ orifice at $0.02 \mathrm{~Pa}$.

\begin{tabular}{|c|c|c|c|c|c|c|}
\hline \multicolumn{3}{|c|}{ component } & \multirow{2}{*}{$\begin{array}{c}\text { weight } \\
1\end{array}$} & \multirow{2}{*}{$\frac{\begin{array}{c}u_{X} \\
(\% ; k=1)\end{array}}{0.143}$} & \multirow{2}{*}{$\frac{\frac{\partial \sigma}{\partial X}}{1}$} & \multirow{2}{*}{$\begin{array}{c}\begin{array}{c}\text { contribution } \\
(\% ; \boldsymbol{k}=\mathbf{1})\end{array} \\
0.14\end{array}$} \\
\hline$u_{\dot{n}}$ & PFM flow rate & $\dot{n}$ & & & & \\
\hline$u_{C_{0}}$ & $\begin{array}{l}11 \mathrm{~mm} \text { orifice } \\
\text { conductance }\end{array}$ & $C_{0}$ & 1 & 0.09 & 1 & 0.09 \\
\hline$u_{\alpha_{1}}$ & $\begin{array}{l}\text { transition flow } \\
\text { pressure coefficient }\end{array}$ & $a_{1}$ & $\alpha_{1} p_{s t d, 0}$ & 2.5 & 0.0024 & 0.01 \\
\hline$u_{R_{P}}$ & pressure ratio & $R_{P}$ & $1 / R_{P}$ & 0.0018 & 0.0382 & 0.01 \\
\hline \multirow{2}{*}{$u_{D C R_{0}}$} & residual drag & $D C R_{0}$ & $D C R_{0}$ & \multirow{2}{*}{$\begin{array}{l}\text { Section } \\
1.9\end{array}$} & \multirow{2}{*}{$\begin{array}{l}\text { Section } \\
1.9\end{array}$} & \multirow{2}{*}{0.03} \\
\hline & & & $\overline{D C R-D C R_{0}}$ & & & \\
\hline$u_{T}$ & temperature & $T$ & $1 / 2$ & 0.05 & 0.5 & 0.03 \\
\hline$u_{B}$ & Type B & & & & & 0.017 \\
\hline \multirow[t]{2}{*}{$u_{A}$} & Type A & $\overline{D C R}$ & 1 & 0.02 & 1 & 0.02 \\
\hline & $\begin{array}{r}\text { accommodation } \\
\text { coefficient }\end{array}$ & $\sigma$ & & & & 0.17 \\
\hline
\end{tabular}


$u_{T}$ : The temperature uncertainty comprises two components: that due to the temperature gradient across the chamber, and that due to the calibration uncertainty. The standard uncertainty associated with the calibration is $20 \mathrm{mK}$ and the typical chamber temperature is $296 \mathrm{~K}$. The standard uncertainty due to the temperature gradient is $0.05 \%$ [32], therefore $u_{T} / 2=0.025 \%$.

\subsection{Uncertainty for the SRG user}

After the SRG has been calibrated at NIST, its uncertainty can only increase. A pressure measurement made with a calibrated SRG will have a relative standard uncertainty $(k=1)$ of

$$
u_{\mathrm{p}, \text { user }}=\left[u_{\sigma}^{2}+\left(u_{T} / 2\right)^{2}+u_{\mathrm{RD}}^{2}+u_{\mathrm{A}}^{2}+u_{L T S}^{2}\right]^{1 / 2} \text {. }
$$

where

$$
\begin{aligned}
u_{\sigma} & =\text { relative standard uncertainty of } \sigma \text { at the time of calibration } \\
u_{T} & =\text { relative standard uncertainty of the user's temperature value } \\
u_{\mathrm{RD}} & =\text { relative standard uncertainty of the residual drag measured by the user } \\
u_{\mathrm{LTS}} & =\text { relative standard uncertainty due to changes of } \sigma \text { after calibration } \\
u_{A} & =\text { Type A relative standard uncertainty of the measurement reproducability }
\end{aligned}
$$

For each contribution, $u$ corresponds to an estimate of the relative standard uncertainty. Additional details of the uncertainty estimates are given below, and the reader is referred to the calibration report for further discussion of Eq. (24). See Ref. [33] for a more general discussion of calculating uncertainties.

$u_{\sigma}$ : An estimate of the relative uncertainty at the time of calibration is given in Table 5 and Section 7.1. This value can change somewhat from calibration to calibration since it depends on the Type A uncertainty, the flow meter that is used, and the exact pressure used to calibrate the gauge. The value of $u_{\sigma}$ is given in the customer calibration report.

$u_{T}$ : The customer will estimate the rotor temperature uncertainty using methods appropriate to their apparatus.

$u_{R D}$ : The estimate of $u_{R D}$ is identical to the quantity given in Eq. (23) in Section 7.1. The customer will use their own values in the formulas.

$u_{L T S}$ : The effective accommodation coefficient will change after the rotor is calibrated.

Removing and re-installing the rotor head, for example, has been shown to change $\sigma$ [22]. If the rotor has a history of NIST calibrations, $u_{\text {LTS }}$ can be estimated from the rotor history. Otherwise, it can be estimated from Table 1 . The average time between calibrations was 2.6 years.

Therefore, the value of $\sigma$ will likely change less than $2 \%$ over a period of two years if the rotor is not physically mishandled or exposed to aggressive gases.

$u_{A}$ : The Type A uncertainty is typically estimated from the standard deviation of the mean of several pressure measurements. Uncertainty in the decrement measurement, $D C R$, is included in the Type A analysis. 


\section{Quality control}

NIST uses the following methods to maintain the quality of the SRG calibration service.

- Adhering to the NIST quality system, which is described briefly in the next section.

- Participating in international comparisons. Four comparisons [34,35,36,37] have been completed since the publication of the previous SP-250-34 [2]. In addition, NIST participated in an SRG key comparison, the CCM.P-K14, during 2010. The draft A of the CCM.P-K-14 was approved in 2014 [38].

- Comparing the medium-range standard to the similar standard used to calibrate ionization chambers. SRGs are used as the transfer standards.

- Comparing the medium-range standard to the NIST primary liquid manometers. SRGs and CDGs are used as the transfer standards.

- Monitoring the SRG check standards whose histories are shown in Figure 5.

- Comparing the flow rates generated by the piston flow meter, the bellows flow meter, and a capillary flow meter that was calibrated independently [39]. The comparison is made by comparing values of $p_{\text {SRG. }}$

\section{NIST quality system}

The calibration services in this publication are supported by the NIST quality system; detailed information and documents regarding the NIST quality system can be found on NIST's website. The integrity, reliability, and traceability of the NIST measurement services relies on the NIST Quality System for Measurement Services (QM-I), which is based on the ISO/IEC 17025 (General requirements for the competence of testing and calibration laboratories) [40] and the relevant requirements of ISO/IEC Guide 34 (General requirements for the competence of reference material producers) [41]. The scope of the NIST Quality System includes the delivery of Calibration Services and the development and certification of Standard Reference Materials.

The NIST quality system for measurement services satisfies the requirements of the International Committee for Weights and Measures (CIPM) Mutual Recognition Arrangement (MRA) [42] for recognition of national measurement standards; and as such, has been recognized as conformant to the ISO/IEC 17025 and ISO Guide 34 by the Inter-American Metrology System (SIM) Quality System Task Force and the Joint Committee of the Regional Metrology Organizations and the BIPM (JCRB). The BIPM is the International Bureau of Weights and Measures.

In order to maintain compliance with the MRA, NIST participates in a large number of international comparisons with other NMIs to support our calibration measurement capabilities and uncertainty claims. Comparisons relevant to the present calibration service can be found by locating the key comparison data base (KCDB) on the BIPM website, and searching on Metrology Area = Mass, Branch = pressure, and Country = United States.

\section{References}

1. C.R. Tilford, S. Dittmann, K.E. McCulloh, "The National Bureau of Standards primary highvacuum standard", J. Vac. Sci. Technol. A 6, 2853-2859 (1988). 
2. S. Dittmann, High vacuum standard and its use, NIST Special Publication SP-250-34, (1989).

3. S. Dittmann, K.E. McCulloh, C.R. Tilford, "Vacuum calibrations using the molecular drag gage", textbook for course taught at NIST (1989).

4. R.W. Hyland, "Operation of the NIST transition range standard", Pressure \& Vacuum Group report (1996).

5. J.P. Looney, "Calculation of the conductance of the orifices used in the NIST primary orifice flow standards", Pressure \& Vacuum Group report (1998).

6. R.F. Chang, "Experimental determination of conductance for mid-range $2 \mathrm{~mm}$ orifice using exponential form", Pressure \& Vacuum Group report (2000).

7. R.F. Berg, "Outgassing in the constant-pressure flow meters used for the vacuum gauge and vacuum leak calibration services", Pressure \& Vacuum Group report (2007).

8. R.F. Berg, "Uncertainties of the constant-pressure flow meters used for the vacuum gauge and vacuum leak calibration services", Pressure \& Vacuum Group report (2007).

9. J.A. Fedchak, "Uncertainty of the pressure ratio", Pressure \& Vacuum Group report (2010).

10. J.A. Fedchak, Conductance and pressure ratio correction factor for the $1 \mathrm{~cm}$ orifice in the midrange vacuum standard", Pressure \& Vacuum Group report (2010).

11. A. Berman, Total Pressure Measurements in Vacuum Technology, Academic Press, New York (1985).

12. K. Jousten, editor, Handbook of Vacuum Technology, Wiley-VCH, Weinheim (2008).

13. W. Jitschin, J. K. Migwit and G. Grosse, "Pressures in the high and medium vacuum range generated by a series expansion standard", Vacuum 40, 293-304 (1990).

14. W. Jitschin, "High-accuracy calibration in the vacuum range $0.3 \mathrm{~Pa}$ to $4000 \mathrm{~Pa}$ using the primary standard of static gas expansion", Metrologia 39, 249-261, (2002).

15. K. Jousten, "Temperature relaxation of argon and helium after injection into a vacuum vessel", Vacuum 45, 1205-1208 (1994).

16. J. K. Fremerey, "Spinning rotor vacuum gauges", Vacuum 32, 685 (1982).

17. S. Dittmann, B. Lindenau, C.R. Tilford, "The molecular drag gauge as a calibration standard", $J$. Vac. Sci. Technol. A 7, 3356-3360 (1989).

18. Choi S-H, Dittman S, Tilford CR, "Stabilization techniques for spinning rotor gage residual drag”, J. Vac. Sci. Technol. A 8, 4079-4085 (1990).

19. K. Jousten, editor, Handbook of Vacuum Technology, pp. 716 - 717, Wiley-VCH, Weinheim (2008).

20. B. E. Lindenau and J. K. Fremerey, "Linearization and temperature compensation up to one atmosphere for the spinning rotor gauge", J. Vac. Sci. Technol. A9, 2737 (1991).

21. J. Setina and J. P. Looney, "Behavior of commercial spinning rotor gages in the transition regime", Vacuum 44, 577- 580 (1993).

22. R.F. Chang and P.J. Abbott, "Factors affecting the reproducibility of the accommodation coefficient of the spinning rotor gauge", J. Vac. Sci. Technol. A 25, 1567-1576 (2007).

23. J. A. Fedchak, K. Arai, K. Jousten, J. Setina, and H. Yoshida, "Recommended Practices for the use of Spinning Rotor Gauges in Inter-laboratory Comparisons", Measurement 66, 176183 (2015).

24. B. E. Lindenau, J. K. Fremerey, and K. Witthauer, "Spinning rotor gauge for operation at free spatial orientation", Vacuum 46, 835 (1995).

25. McCulloh KE, Tilford CR, Ehrlich CD, Long FG, "Low-range flowmeters for use with vacuum and leak standards", Journal of Vacuum Science \& Technology A 5, 376-381 (1987). 
26. A.W. Hartman, "Report of measurement: Measuring specified dimensions of two supplied rings", NIST memorandum to J.P. Looney (1990).

27. R.F. Chang, "Experimental determination of conductance for mid-range $2 \mathrm{~mm}$ orifice using polynomial form", Pressure \& Vacuum Group report (2000).

28. F. Sharipov, "Numerical simulation of rarefied gas flow through a thin orifice", J. Fluid Mech. 518, 35-60 (2004)

29. G. Comsa, J.K. Fremerey, B. Lindenau, G. Messer, P. Röhl, "Calibration of a spinning rotor gas friction gauge against a fundamental vacuum pressure standard”, J. Vac. Sci. Technol. A 17, 642-644 (1980).

30. J. K. Fremerey, “The spinning rotor gauge”, J. Vac. Sci. Technol. A 3, 1715-1720 (1985).

31. J. Šetina, "Two point calibration scheme for the linearization of the spinning roter gauge at transition regime pressures", J. Vac. Sci. Technol. A 17, 2086-2092 (1999).

32. J. A. Fedchak, "Uncertainty due to Temperature Gradient in the UHV chamber", Pressure \& Vacuum Group Report (2011).

33. B.N. Taylor and C.E. Kuyatt, Guidelines for evaluating and expressing the uncertainty of NIST measurement results, NIST Technical Note 1297 (1994). Available at physics.nist.gov/Pubs/guidelines/TN1297/tn1297s.pdf.

34. G. Messer, W. Jitschin L. Rubet, A. Calcatelli, F.J. Redgrave, A. Keprt, W. Fei, J.K.N. Sharma, S. Dittmann, M. Ono. "Intercomparison of 9 national high-vacuum standards under the auspices of the Bureau International des Poids et Mesures", Metrologia 26, 183-195 (1989).

35. K. Jousten, A.R. Filippelli, C.R. Tilford, F.J. Redgrave, "Comparison of the standards for high and ultrahigh vacuum at three national standards laboratories", J. Vac. Sci. Technol. A 15, 2395-2406 (1997).

36. D.A. Olson, P.J. Abbott, K. Jousten, F.J. Redgrave., P. Mohan, S.S. Hong, "Final report of key comparison CCM.P-K3: absolute pressure measurements in gas from $3 \times 10^{-6} \mathrm{~Pa}$ to $9 \times 10^{-4} \mathrm{~Pa} "$, Metrologia 47, 07004 (2010).

37. "Final Report of the Bilateral key comparison CCM.P-K3.1 for absolute pressure measurements from $3 \times 10^{-6} \mathrm{~Pa}$ to $9 \times 10^{-4} \mathrm{~Pa}$ " J. A. Fedchak, Th. Bock, and K. Jousten, Metrologia Tech. Suppl. 51, 07005 (2014).

38. "Draft A report on the key comparison, CCM.M.P-K14 in the pressure range from $1 \times 10^{-4} \mathrm{~Pa}$ to 1.0 Pa", C. Wuethrich, H. Akimichi, M. Bergoglio, J. A. Fedchak, K. Jousten5, S. Soo Hong, J. T. Guzman, Draft A approved 2013, Draft B in progress.

39. R.F. Berg, "Capillary flow meter for calibrating spinning rotor gauges", J. Vac. Sci. Technol. A 26, 1161-1165 (2008).

40. ISO/IEC 17025:2005(E), "General requirements for the competence of testing and calibration laboratories" (2005).

41. ISO Guide 34:2000(E), "General requirements for the competence of reference material producers" (2000).

42. CIPM, "Mutual recognition of national measurement standards and of calibration and measurement certificates issued by national metrology institutes", www.bipm.org/utils/en/pdf/mra_2003.pdf, (1999, rev. 2003). 
11. Appendix: Example calibration report

\author{
NLT \\ National Institute of Standards and Technology \\ Technology Administration, U.S. Department of Commerce
}

\title{
REPORT OF CALIBRATION
}

Spinning Rotor Vacuum Gauge (service ID no. 30030C)

11 June 2014

$\begin{array}{ll}\text { NIST test folder no. } & 285 \times X X-14 \\ \text { customer purchase order no. } & \text { NN1234 } \\ \text { rotor D } & 191459 \\ \text { SRG database no. } & \text { MR-241 }\end{array}$

Gauge submitted by:

Anony Mouse

NoName, Inc.

1 Way St.

Washington DC, 20002

Gauge calibrated by:

\author{
James Fedchak \\ Thermodynamic Metrology Group \\ Sensor Science Division \\ National Institute of Standards and Technology \\ Gaithersburg, MD 20899-8364
}




\section{Summary}

\section{Test dates}

29 May, 2014 to 09 July, 2014

Spinning rotor gauge identification

\begin{tabular}{rcc}
\multicolumn{3}{c}{ Spinning rotor gauge identification } \\
\hline & model & serial no. \\
\hline rotor & & 191459 \\
head & MKS SRG-SH700 & $94080 \mathrm{G}$ \\
controller & MKS SRG-2CE & 5000531 \\
\hline
\end{tabular}

Calibration parameters

\begin{tabular}{lc}
\hline mean effective accommodation coefficient $\sigma$ & 0.9650 \\
rotor diameter $(\mathrm{mm})$ & 4.493 \\
rotor density $\left(\mathrm{g} / \mathrm{cm}^{3}\right)$ & 7.806 \\
test gas & nitrogen \\
test gas molecular weight $(\mathrm{g} / \mathrm{mol})$ & 28.013 \\
sampling interval & $30 \mathrm{~s}$ \\
upper speed & $415 \mathrm{~Hz}$ \\
lower speed & $405 \mathrm{~Hz}$ \\
\hline
\end{tabular}

Comparison with last calibration

\begin{tabular}{lc}
\hline date of previous calibration & $5-\operatorname{Dec} 2011$ \\
previous accommodation coefficient & 1.0159 \\
change from previous value (\%) & -5.0 \\
\hline
\end{tabular}

Uncertainty of the effective accommodation coefficient $\sigma$ (See text for more detail.)

relative standard uncertainty of the mean $D C R$ reading at NIST

relative standard uncertainty of the pressure $P_{\text {std }}$ by the standard

\begin{tabular}{ll}
$u_{\text {mean }, \text { NST }}$ & $0.014 \%$ \\
$u_{\mathrm{P}}$ & $0.189 \%$ \\
$u_{\sigma}$ & $0.190 \%$ \\
$U_{\sigma}$ & $0.38 \%$ \\
\hline
\end{tabular}

relative total standard $(k=1)$ uncertainty of $\sigma$ at the time of calibration

relative total expanded $(k=2)$ uncertainty of $\sigma$ at the time of calibration

$u_{\sigma}$

Page 2 of 8

Test folder $285 \mathrm{XXX}-14$ 


\section{Test equipment}

The rotor was calibrated with the NIST primary transition-range vacuum standard, which generates a known pressure by sending gas at a known flow rate through an orifice of known area. A detailed description of a similar standard can be found in reference [1]. For a customer not providing a controller and suspension head (Service ID no. 30029C), a NISTowned controller and suspension head also were used.

\section{Preparation}

The rotor was mounted in its thimble, and the thimble was mounted onto the vacuum chamber, which included three NIST check standard spinning rotor gauges. Other customer spinning rotor gauges also were mounted onto the chamber.

\section{Data collection and analysis}

Prior to the calibration runs the pressure-independent offset, also known as the residual drag, was measured while the vacuum chamber was at its base pressure, typically about $10^{-6} \mathrm{~Pa}$. The offset depended on the rotor frequency $f$, and this dependence was measured and used to correct the measured offset to its value at the reference frequency, $f=410 \mathrm{~Hz}$. All calibration data were subsequently corrected for this pressure-independent offset.

A total of 12 calibration runs were performed on three days. Each run comprised repeated measurements of the normalized rotor deceleration rate $(D C R)$ while nitrogen flowed from the NIST piston flow meter into the vacuum chamber to generate a known pressure $P_{\text {std. }}$ The typical flow rate was $0.1 \mu \mathrm{mol} / \mathrm{s}$, and the typical chamber pressure was $0.02 \mathrm{~Pa}$. The recorded values of temperature $(\mathrm{K})$ and $D C R\left(\mathrm{~s}^{-1}\right)$ were converted into values of pressure $P_{\mathrm{SRG}}(\mathrm{Pa})$ as follows [2].

$$
P_{\mathrm{SRG}}=\left(\frac{2 \pi R T}{M}\right)^{1 / 2} \frac{\rho d}{10}\left(D C R-D C R_{0}\right)
$$

where

$$
\begin{aligned}
R & =\text { universal gas constant }\left(8.314772 \mathrm{~J} \mathrm{~mol}^{-1} \mathrm{~K}^{-1}\right) \\
T & =\text { gas temperature }(\mathrm{K}) \\
M & =\text { gas molar mass }\left(\mathrm{kg} \mathrm{mol}{ }^{-1}\right) \\
\rho & =\text { rotor density }\left(\mathrm{kg} \mathrm{m}^{-3}\right) \\
d & =\text { rotor diameter }(\mathrm{m}) \\
D C R & =-(\dot{f} / f)=\text { normalized rotor deceleration rate }\left(\mathrm{s}^{-1}\right) \\
D C R_{0} & =\text { offset }=\text { value of } D C R \text { measured at base pressure }(\mathrm{Pa})
\end{aligned}
$$

The rotor was then characterized by the effective accommodation coefficient defined by the pressure ratio

$$
\sigma=\frac{P_{\mathrm{SRG}}}{P_{\mathrm{std}}}
$$


This value is termed "effective" because it is calculated using the nominal values of the rotor diameter and density listed on the summary page.

Averaging the calibration runs and using the rotor diameter and density given on the summary page yielded the mean effective accommodation coefficient $\sigma$. This value is valid for pressures less than $0.1 \mathrm{~Pa}$. A table and graph of previous NIST calibration results, if any, is included at the end of the report.

\section{Uncertainty}

A pressure measurement made with this gauge will have a relative standard uncertainty of

$$
U_{\mathrm{p}, \text { user }}=2\left[u_{\sigma}^{2}+\left(u_{T} / 2\right)^{2}+u_{\mathrm{RD}}^{2}+u_{\mathrm{A}}^{2}+u_{L T S}^{2}\right]^{1 / 2} \text {. }
$$

where

$u_{\sigma}=$ relative standard uncertainty of $\sigma$ at the time of calibration

$u_{T}=$ relative standard uncertainty of the user's temperature value

$u_{R D}=$ relative standard uncertainty of the residual drag measured by the user

$u_{A}=$ Type A relative standard uncertainty of the measurement reproducability

$u_{L T S}=$ relative standard uncertainty due to changes of $\sigma$ after calibration

For each contribution, the standard uncertainty $u$ corresponds to an estimated standard deviation. The expanded uncertainty $U_{p}$ corresponds to a coverage factor $k=2$, which means that if the user measures a value $P_{\text {user }}=P_{\mathrm{SRC}} / \sigma$, the true pressure has a $95 \%$ probability of lying within the interval $P_{\mathrm{SRG}}\left(1 \pm U_{P}\right)$. More detail about the component uncertainties is given below. See Ref. [3] for a more general discussion of calculating uncertainty.

$u_{\sigma}$ is the total standard uncertainty of $\sigma$ at the time of calibration. The value of $u_{\sigma}$ is given on the first page of this report.

$u_{T}$ is the relative standard uncertainty of the temperature of the user's vacuum chamber. The following example illustrates the significance of this component: Suppose that the user enters into the controller a value that is in error by $\Delta T=1 \mathrm{~K}$. Equation (1) then implies a corresponding pressure error of $(\Delta T / T) / 2=0.17 \%$.

$u_{\mathrm{A}}$ is the relative standard uncertainty of the mean value of $D C R$ or pressure due to random variation of the readings. For example, for $n$ readings of the pressure $P$, it is the estimated standard deviation of the mean given by

$$
u_{A}=\left[\frac{1}{n(n-1)} \sum_{k=1}^{n}\left(P_{k}-\langle P\rangle\right)^{2}\right]^{1 / 2},
$$

where $\langle P\rangle$ is the average value. This component is a "Type $\mathrm{A}$ " uncertainty because it is evaluated by statistical means [3]. Uncertainty in the decrement measurement, $D C R$, is included in the Type A analysis. 
The value of $u_{\mathrm{A}}$ will depend on the strength of the rotor's rotational timing signal, the measurement time interval, local temperature instabilities, and local vibration levels. The user should evaluate $u_{\mathrm{A}}$ by making repeated measurements at a stable pressure.

$u_{R D}$ is the relative standard uncertainty of the residual drag or pressure offset. This component can be estimated from the expression:

$$
u_{R D}=\frac{\Delta D C R_{0}}{D C R-D C R_{0}}=\frac{\Delta P_{S R G, 0}}{P_{S R G}} .
$$

Thus there are two equivalent methods of estimating $u_{R D}: 1$ ) divide the absolute reproducibility of the residual drag decrement, $\triangle D C R_{0}$, by the net decrement above the offset at the pressure of interest $D C R-D C R_{0}$, or 2) divide the absolute reproducibility of the residual drag in units of pressure, $\triangle P_{S R G, 0}$, by the measured pressure of interest $P_{S R G \text {. }}$

$u_{L T S}$ is the relative standard uncertainty due to the long-term stability of the accommodation coefficient. The accommodation coefficient will change after the calibration. Reference [4] discusses factors that affect the reproducibility of the accommodation coefficient, of which the most important is the removal and reinstallation of the gauge head.

If the results of previous NIST calibrations are included at the end of this report, they can be used to estimate $u_{\text {change. }}$. If the results of previous NIST calibrations are not available, $u_{\text {change }}$ can be estimated from the following statistics from our database of spinning rotor gauge calibrations, which spans more than 20 years. The change between calibrations was less than $0.5 \%$ for $41 \%$ of the time, less than $1 \%$ for $63 \%$ of the time, and less than $2 \%$ for $86 \%$ of the time. The average time between calibrations was 2.6 years.

Therefore, the value of $\sigma$ will likely change less than $2 \%$ over a period of two years if the rotor is not physically mishandled or exposed to aggressive gases.

\section{Comments}

This calibration does not apply at pressure greater than $0.1 \mathrm{~Pa}$. At lower pressures we expect the effective accommodation coefficient to be primarily a function of the gas-surface interactions, which depend on the characteristics of the rotor surface. However, at pressures greater than $0.1 \mathrm{~Pa} \sigma$ may depend also on the dimensions of the rotor and its thimble, and possibly on the particular head and controller used.

This calibration applies only to the test gas, nitrogen, with at least $99.9 \%$ purity. Also, it must be used in conjunction with the calibration parameters given on the summary page. External magnetic fields (time-dependent ones in particular) may have an unpredictable effect on the behavior of the gauge.

Exercise care when handling the rotor. It should be touched only with clean plastic gloves or clean, nonmagnetic tools such as plastic or plastic-covered stainless steel 
tweezers. Touching the rotor with bare fingers will lead to surface corrosion and a subsequent change in the accommodation coefficient. Avoid other causes of surface damage, such as exposure to pump oil.

Enter, with the appropriate units, the necessary parameters into the controller. In addition to the effective accommodation coefficient $\sigma$, these include values of the rotor diameter and density that were used in this calibration.

Determine the offset correction and its frequency dependence at the time of use. The customer must determine and properly account for these parameters because the magnitude and frequency dependence of the offset correction may change at any time after the inductive (motor) drive circuit has been activated, particularly if the rotor has been unsuspended and suspended. The offset correction and its frequency dependence are determined by recording the indicated pressure readings at base pressure as a function of frequency.

Always stop the rotation before unsuspending the rotor in its thimble.

Inspect the rotor periodically. Changes in its appearance, for example, new scratches, corrosion, or pitting, may indicate that the calibration is no longer valid.

Consider adding a second spinning rotor gauge to the vacuum system. Comparing the two gauges can quantify any change of $\sigma$ due to accidental mishandling of one of the rotors. 


\section{References}

1. S. Dittmann, High vacuum standard and its use, NIST Special Publication SP-250-34, (1989).

2. J.K. Fremerey, "The spinning rotor gauge", J. Vac. Sci. Technol. A 3, 1715-1720 (1985).

3. B.N. Taylor and C.E. Kuyatt, Guidelines for evaluating and expressing the uncertainty of NIST measurement results, NIST Technical Note 1297 (1994). Available at physics.nist.gov/Pubs/guidelines/TN1297/tn1297s.pdf.

4. R.F. Chang and P.J. Abbott, "Factors affecting the reproducibility of the accommodation coefficient of the spinning rotor gauge", J. Vac. Sci. Technol. A 25, 1567-1576 (2007). 
History of NIST calibration of rotor ID number 191459

\begin{tabular}{lccccc}
\hline \multirow{2}{*}{ start date } & \multirow{2}{*}{ NIST ref. } & $\begin{array}{c}\text { ref. diameter ref. density } \\
(\mathbf{m m})\end{array}$ & $\begin{array}{c}\text { gas } \\
\left(\mathbf{g} / \mathbf{c m}^{\mathbf{3}}\right)\end{array}$ & $\begin{array}{c}\text { accommodation } \\
\text { coefficient } \boldsymbol{\sigma}\end{array}$ \\
\hline 16-Jan-2003 & MR-119 & 4.491 & 7.823 & $\mathrm{~N}_{2}$ & 1.0701 \\
6-Oct-2005 & MR-142 & 4.491 & 7.823 & $\mathrm{~N}_{2}$ & 0.9993 \\
11-Dec-2008 & MR-178 & 4.491 & 7.823 & $\mathrm{~N}_{2}$ & 0.9808 \\
5-Dec-2011 & MR-208 & 4.491 & 7.823 & $\mathrm{~N}_{2}$ & 1.0159 \\
4-Jun-2014 & MR-241 & 4.491 & 7.823 & $\mathrm{~N}_{2}$ & 0.9650 \\
\hline
\end{tabular}

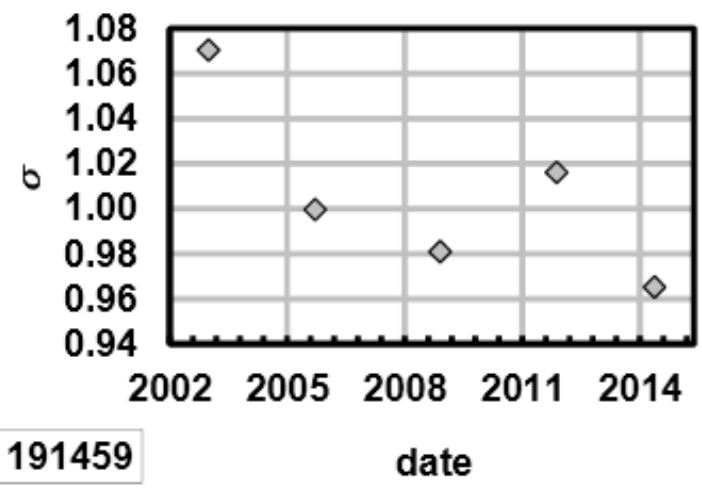

For the Director of the National Institute of Standards and Technology,

Gregory F. Strouse

Sensor Science Division

Leader, Thermodynamic Metrology Group 